DPSU-13-4

\title{
Equivalences of the Multi-Indexed Orthogonal Polynomials
}

\author{
Satoru Odake \\ Department of Physics, Shinshu University, \\ Matsumoto 390-8621, Japan
}

\begin{abstract}
Multi-indexed orthogonal polynomials describe eigenfunctions of exactly solvable shape-invariant quantum mechanical systems in one dimension obtained by the method of virtual states deletion. Multi-indexed orthogonal polynomials are labeled by a set of degrees of polynomial parts of virtual state wavefunctions. For multi-indexed orthogonal polynomials of Laguerre, Jacobi, Wilson and Askey-Wilson types, two different index sets may give equivalent multi-indexed orthogonal polynomials. We clarify these equivalences. Multi-indexed orthogonal polynomials with both type I and II indices are proportional to those of type I indices only (or type II indices only) with shifted parameters.
\end{abstract}

\section{Introduction}

Exactly solvable quantum mechanical systems in one dimension have seen remarkable developments in recent years [1]-30. Important ingredients are new types of orthogonal polynomials; exceptional orthogonal polynomials [1, 3, 23, 25] and its generalization, multiindexed orthogonal polynomials [4, 5, 26, 27]. Multi-indexed orthogonal polynomials are the main parts of eigenfunctions of the shape-invariantly deformed quantum mechanical systems, which are obtained from the original shape-invariant systems by the method of virtual states deletion. Multi-index orthogonal polynomials of Laguerre, Jacobi, Wilson, Askey-Wilson, Racah and $q$-Racah types have been constructed. They satisfy second order differential/difference equations and form a complete set. Their degrees start from some positive integer $\ell$ and Bochner's theorem [31] and its generalization are avoided. 
The method of virtual states deletion [5] is a systematic procedure to obtain iso-spectral exactly solvable quantum mechanical systems from the original exactly solvable systems. It based on the Darboux-Crum transformation [32, 33] taking virtual state wavefunctions as seed solutions. The Darboux-Crum transformation in terms of eigenfunctions gives KreinAdler transformation [34], by which some eigenstates of the original system are deleted. The Darboux-Crum transformation in terms of pseudo virtual state wavefunctions [18, 30] gives a deformed system in which some extra eigenstates are added to the original system. Virtual and pseudo virtual state wavefunctions are obtained from the eigenfunctions by using the discrete symmetries of the Hamiltonian [5, 26, 27, 18, 30].

Multi-indexed orthogonal polynomials are labeled by an index set $\mathcal{D}=\left\{d_{1}, \ldots, d_{M}\right\}$, where $d_{j}$ is the degree of the polynomial part of the virtual state wavefunction for deletion. For Laguerre, Jacobi, Wilson and Askey-Wilson cases, we have two types of virtual states, type I and type II [5, 27]. For these cases, it may happen that the deformed system labeled by an index set $\mathcal{D}$ and another deformed system labeled by a different index set $\mathcal{D}^{\prime}$ with shifted parameters are equivalent, which means that the corresponding two multiindexed orthogonal polynomial labeled by $\mathcal{D}$ and $\mathcal{D}^{\prime}$ (with shifted parameters) are proportional. In [5, 27], we have presented such examples, $\mathcal{D}=\left\{m^{\mathrm{I}},(m+1)^{\mathrm{I}}, \ldots,(m+k-1)^{\mathrm{I}}\right\}$ and $\mathcal{D}^{\prime}=\left\{k^{\mathrm{II}},(k+1)^{\mathrm{II}}, \ldots,(k+m-1)^{\mathrm{II}}\right\}$. A certain extension of this result has been commented in [18, 30], where Wronskian/Casoratian identities and equivalence of two deformed systems are obtained by using the Darboux-Crum transformation in terms of pseudo virtual state wavefunctions. In this paper we generalize this result. By extending the Wronskian/Casoratian identities given in [18, 30], we show that the multi-indexed orthogonal polynomials with both type I and II indices (2.11) are proportional to those with type I indices only (4.11) (or type II indices only (4.16)).

This paper is organized as follows. The Darboux-Crum approach to quantum mechanical systems and the multi-indexed orthogonal polynomials is recapitulated in section 2, After recalling the Wronskian/Casoratian identities, we extend them in section 3 . Section 4 is the main part of the paper. By using the Wronskian/Casoratian identities obtained in $\S 3$, we show the equivalence of two deformed systems obtained by the Darboux-Crum transformation in terms of virtual state wavefunctions; one uses both type I and II virtual states and the other uses type I only (or type II only) with shifted parameters. This implies that multiindexed orthogonal polynomials with both type I and II indices are proportional to those 
with type I indices only (or type II indices only) with shifted parameters. The final section is for a summary and comments. Various data of quantum mechanical systems described by Laguerre, Jacobi, Wilson and Askey-Wilson polynomials are presented in Appendix.

\section{Quantum Mechanical Systems and Multi-Indexed Orthogonal Polynomials}

Our analysis is based on the quantum mechanical formulation, in which the Schrödinger equation is a second order differential (oQM, ordinary Quantum Mechanics) or difference (idQM, discrete Quantum Mechanics with pure imaginary shifts). See, for example, [22] for the general introduction. Here we recapitulate the Darboux-Crum approach to quantum mechanical systems with a continuous dynamical variable $x$ and the multi-indexed orthogonal polynomials [32, 33, 34, 20, 21, [5, 27].

We consider quantum mechanical systems in one dimension $\left(x_{1}<x<x_{2}\right)$,

$$
\begin{aligned}
& \mathcal{H}=\mathcal{A}^{\dagger} \mathcal{A} \\
& \quad= \begin{cases}p^{2}+U(x) & : \text { oQM } \\
\sqrt{V(x)} e^{\gamma p} \sqrt{V^{*}(x)}+\sqrt{V^{*}(x)} e^{-\gamma p} \sqrt{V(x)}-V(x)-V^{*}(x) & : \text { idQM }\end{cases} \\
& \mathcal{H} \phi_{n}(x)=\mathcal{E}_{n} \phi_{n}(x) \quad\left(n \in \mathbb{Z}_{\geq 0}\right), \quad 0=\mathcal{E}_{0}<\mathcal{E}_{1}<\mathcal{E}_{2}<\cdots, \\
& \left(\phi_{n}, \phi_{m}\right) \stackrel{\text { def }}{=} \int_{x_{1}}^{x_{2}} d x \phi_{n}(x)^{*} \phi_{m}(x)=h_{n} \delta_{n m} \quad\left(0<h_{n}<\infty\right),
\end{aligned}
$$

where the momentum operator is $p=-i \frac{d}{d x}$ and $\gamma$ is a real number. The explicit forms of $\mathcal{A}$ and $\mathcal{A}^{\dagger}$ are given in Appendix. Any solution of the Schrödinger equation $\mathcal{H} \tilde{\varphi}(x)=$ $\tilde{\mathcal{E}} \tilde{\varphi}(x)$, which needs not be square integrable, can be used as a seed solution for the Darboux transformation. The Hamiltonian $\mathcal{H}$ can be written as

$$
\mathcal{H}=\hat{\mathcal{A}}_{\tilde{\varphi}}^{\dagger} \hat{\mathcal{A}}_{\tilde{\varphi}}+\tilde{\mathcal{E}}, \quad \hat{\mathcal{A}}_{\tilde{\varphi}} \tilde{\varphi}(x)=0
$$

and the Darboux transformation is

$$
\mathcal{H} \rightarrow \mathcal{H}^{\prime}=\hat{\mathcal{A}}_{\tilde{\varphi}} \hat{\mathcal{A}}_{\tilde{\varphi}}^{\dagger}+\tilde{\mathcal{E}}, \quad \psi(x) \rightarrow \psi^{\prime}(x)=\hat{\mathcal{A}}_{\tilde{\varphi}} \psi(x) .
$$

Then it is easy to show

$$
\mathcal{H} \psi(x)=\mathcal{E} \psi(x) \Rightarrow \mathcal{H}^{\prime} \psi^{\prime}(x)=\mathcal{E} \psi^{\prime}(x)
$$


Therefore we have $\mathcal{H}^{\prime} \phi_{n}^{\prime}(x)=\mathcal{E}_{n} \phi_{n}^{\prime}(x)$. The state corresponding to the used seed solution is deleted, $\tilde{\varphi}^{\prime}(x)=0$. This procedure can be repeated. Problems are (a) the deformed system $\mathcal{H}^{\prime}$ is non-singular or not, (b) there exist extra eigenstates other than $\phi_{n}^{\prime}(x)$ or not. Under the assumption that the deformed system is non-singular, answer to (b) depends on the choice of seed solutions. There are three cases: (i)[state deleting] some eigenstates are deleted, (ii)[state adding] some eigenstates are added, (iii)[iso-spectral] no eigenstates are deleted or added. We know several types of seed solutions, for example,

$$
\begin{array}{ll}
\tilde{\varphi}(x)=\phi_{n}(x) \quad \text { : eigenstate } & \Rightarrow \text { state deleting, } \\
\tilde{\varphi}(x)=\tilde{\phi}_{\mathrm{v}}^{\text {pvs }}(x): \text { pseudo virtual state } & \Rightarrow \text { state adding, } \\
\tilde{\varphi}(x)=\tilde{\phi}_{\mathrm{v}}^{\mathrm{vs}}(x): \text { virtual state } & \Rightarrow \text { iso-spectral. }
\end{array}
$$

Virtual and pseudo virtual state wavefunctions are obtained from eigenfunctions by using the discrete symmetry of the Hamiltonian. For their definition, see [5, 27, 18, 30]. After multistep Darboux transformations, various quantities are expressed in terms of the Wronskian $\mathrm{W}[\cdots]$ or Casoratian $\mathrm{W}_{\gamma}[\cdots]$,

$$
\begin{aligned}
& \mathrm{W}\left[f_{1}, f_{2}, \ldots, f_{n}\right](x) \stackrel{\text { def }}{=} \operatorname{det}\left(\frac{d^{j-1} f_{k}(x)}{d x^{j-1}}\right)_{1 \leq j, k \leq n}, \\
& \mathrm{~W}_{\gamma}\left[f_{1}, f_{2}, \ldots, f_{n}\right](x) \stackrel{\text { def }}{=} i^{\frac{1}{2} n(n-1)} \operatorname{det}\left(f_{k}\left(x_{j}^{(n)}\right)\right)_{1 \leq j, k \leq n}, \quad x_{j}^{(n)} \stackrel{\text { def }}{=} x+i\left(\frac{n+1}{2}-j\right) \gamma,
\end{aligned}
$$

and $\mathrm{W}[\cdot](x)=\mathrm{W}_{\gamma}[\cdot](x)=1$ for $n=0$. For example, after $s$-step (seed solutions $\tilde{\varphi}_{1}(x), \ldots$, $\tilde{\varphi}_{s}(x)$ are used in this order), eigenfunctions $\phi_{n}^{[s]}(x)$ with energy $\mathcal{E}_{n}$ are

$$
\begin{aligned}
\mathrm{oQM}: \quad \phi_{n}^{[s]}(x)= & \frac{\mathrm{W}\left[\tilde{\varphi}_{1}, \ldots, \tilde{\varphi}_{s}, \phi_{n}\right](x)}{\mathrm{W}\left[\tilde{\varphi}_{1}, \ldots, \tilde{\varphi}_{s}\right](x)}, \\
\operatorname{idQM~:~} \phi_{n}^{[s]}(x)= & \left(\frac{\sqrt{\prod_{j=0}^{s-1} V\left(x+i\left(\frac{s}{2}-j\right) \gamma\right) V^{*}\left(x-i\left(\frac{s}{2}-j\right) \gamma\right)}}{\mathrm{W}_{\gamma}\left[\tilde{\varphi}_{1}, \ldots, \tilde{\varphi}_{s}\right]\left(x-i \frac{\gamma}{2}\right) \mathrm{W}_{\gamma}\left[\tilde{\varphi}_{1}, \ldots, \tilde{\varphi}_{s}\right]\left(x+i \frac{\gamma}{2}\right)}\right)^{\frac{1}{2}} \\
& \times \mathrm{W}_{\gamma}\left[\tilde{\varphi}_{1}, \ldots, \tilde{\varphi}_{s}, \phi_{n}\right](x) .
\end{aligned}
$$

Multi-step Darboux transformation in terms of eigenfunctions only (say case (A)) gives the Crum's theorem [33, 20] and its generalization, Krein-Adler transformation [34, 21]. That of pseudo virtual state wavefunctions only (say case (B)) leads to Wronskian/Casoratian identities [18, 30], which implies equivalence of case (B) and case (A) with shifted parameters. That of virtual state wavefunctions only presents the multi-indexed orthogonal polynomials [5, 27]. 
The quantum systems to be considered have some parameters (coupling constants), denoted symbolically by $\boldsymbol{\lambda}=\left(\lambda_{1}, \lambda_{2}, \ldots\right), \mathcal{H}=\mathcal{H}(\boldsymbol{\lambda}), \mathcal{E}_{n}=\mathcal{E}_{n}(\boldsymbol{\lambda}), \phi_{n}(x)=\phi_{n}(x ; \boldsymbol{\lambda}), P_{n}(\eta)=$ $P_{n}(\eta ; \boldsymbol{\lambda})$, etc. Various data of quantum mechanical systems described by Laguerre(L), Jacobi $(J)$, Wilson(W) and Askey-Wilson(AW) polynomials are given in Appendix.

The multi-indexed orthogonal polynomials of L, J, W and AW types are the main part of the eigenfunctions of the deformed systems obtained from the original systems by the method of virtual states deletion. There are two types of virtual states [5, 27], type I $\tilde{\phi}_{\mathrm{v}}^{\mathrm{I}}(x)$ and type II $\tilde{\phi}_{\mathrm{v}}^{\mathrm{II}}(x)\left(\mathrm{v} \in \mathbb{Z}_{\geq 0}\right)$, which are derived by the discrete symmetries of the original Hamiltonian and $\mathrm{v}$ denotes the degree of polynomial part. The set of virtual state wavefunctions for deletion is characterized by their degrees:

$$
\begin{aligned}
\mathcal{D}= & \left\{d_{1}, \ldots, d_{M}\right\}=\left\{d_{1}^{\mathrm{I}}, \ldots, d_{M_{\mathrm{I}}}^{\mathrm{I}}, d_{1}^{\mathrm{II}}, \ldots, d_{M_{\mathrm{II}}}^{\mathrm{II}}\right\} \quad\left(M_{\mathrm{I}}, M_{\mathrm{II}} \geq 0, M=M_{\mathrm{I}}+M_{\mathrm{II}}\right), \\
& \left(d_{j}^{\mathrm{I}} \in \mathbb{Z}_{\geq 0}, \text { mutually distinct } ; d_{j}^{\mathrm{II}} \in \mathbb{Z}_{\geq 0}, \text { mutually distinct }\right),
\end{aligned}
$$

namely we use seed solutions

$$
\tilde{\phi}_{d_{1}^{\mathrm{I}}}^{\mathrm{I}}(x ; \boldsymbol{\lambda}), \ldots, \tilde{\phi}_{d_{M_{\mathrm{I}}}^{\mathrm{I}}}^{\mathrm{I}}(x ; \boldsymbol{\lambda}), \tilde{\phi}_{d_{1}^{\mathrm{II}}}^{\mathrm{II}}(x ; \boldsymbol{\lambda}), \ldots, \tilde{\phi}_{d_{M_{\mathrm{II}}}^{\mathrm{II}}}^{\mathrm{II}}(x ; \boldsymbol{\lambda}) .
$$

The deformed systems are [5, 27]

$$
\mathcal{H}_{\mathcal{D}}(\boldsymbol{\lambda}) \phi_{\mathcal{D} n}(x ; \boldsymbol{\lambda})=\mathcal{E}_{n}(\boldsymbol{\lambda}) \phi_{\mathcal{D} n}(x ; \boldsymbol{\lambda}) \quad(n=0,1, \ldots) .
$$

Here the Hamiltonians of the deformed systems are

$$
\begin{aligned}
& \mathrm{L}, \mathrm{J}: \quad \mathcal{H}_{\mathcal{D}}(\boldsymbol{\lambda})=p^{2}+U_{\mathcal{D}}(x ; \boldsymbol{\lambda}), \quad U_{\mathcal{D}}(x ; \boldsymbol{\lambda})=\frac{\partial_{x}^{2} \phi_{\mathcal{D} 0}(x ; \boldsymbol{\lambda})}{\phi_{\mathcal{D} 0}(x ; \boldsymbol{\lambda})}, \\
& \mathrm{W}, \mathrm{AW}: \quad \mathcal{H}_{\mathcal{D}}(\boldsymbol{\lambda})= \sqrt{V_{\mathcal{D}}(x ; \boldsymbol{\lambda})} e^{\gamma p} \sqrt{V_{\mathcal{D}}^{*}(x ; \boldsymbol{\lambda})}+\sqrt{V_{\mathcal{D}}^{*}(x ; \boldsymbol{\lambda})} e^{-\gamma p} \sqrt{V_{\mathcal{D}}(x ; \boldsymbol{\lambda})} \\
&-V_{\mathcal{D}}(x ; \boldsymbol{\lambda})-V_{\mathcal{D}}^{*}(x ; \boldsymbol{\lambda}), \\
& V_{\mathcal{D}}(x ; \boldsymbol{\lambda})=V\left(x ; \boldsymbol{\lambda}^{\left[M_{\mathrm{I}}, M_{\mathrm{II}}\right]}\right) \frac{\check{\Xi}_{\mathcal{D}}\left(x+i \frac{\gamma}{2} ; \boldsymbol{\lambda}\right)}{\check{\Xi}_{\mathcal{D}}\left(x-i \frac{\gamma}{2} ; \boldsymbol{\lambda}\right)} \frac{\check{\Xi}_{\mathcal{D}}(x-i \gamma ; \boldsymbol{\lambda}+\boldsymbol{\delta})}{\check{\Xi}_{\mathcal{D}}(x ; \boldsymbol{\lambda}+\boldsymbol{\delta})},
\end{aligned}
$$

and their eigenfunctions are

$$
\begin{aligned}
& \phi_{\mathcal{D} n}(x ; \boldsymbol{\lambda}) \propto \psi_{\mathcal{D}}(x ; \boldsymbol{\lambda}) \check{P}_{\mathcal{D}, n}(x ; \boldsymbol{\lambda}), \\
& \psi_{\mathcal{D}}(x ; \boldsymbol{\lambda})=\phi_{0}\left(x ; \boldsymbol{\lambda}^{\left[M_{\mathrm{I}}, M_{\mathrm{II}}\right]}\right) \times \begin{cases}\check{\Xi}_{\mathcal{D}}(x ; \boldsymbol{\lambda})^{-1} & : \mathrm{L}, \mathrm{J} \\
\left(\check{\Xi}_{\mathcal{D}}\left(x-i \frac{\gamma}{2} ; \boldsymbol{\lambda}\right) \check{\Xi}_{\mathcal{D}}\left(x+i \frac{\gamma}{2} ; \boldsymbol{\lambda}\right)\right)^{-\frac{1}{2}} & : \mathrm{W}, \mathrm{AW}\end{cases} \\
& \check{P}_{\mathcal{D}, 0}(x ; \boldsymbol{\lambda}) \propto \check{\Xi}_{\mathcal{D}}(x ; \boldsymbol{\lambda}+\boldsymbol{\delta}),
\end{aligned}
$$


where $\boldsymbol{\lambda}^{\left[M_{\mathrm{I}}, M_{\mathrm{II}}\right]}$ is

$$
\boldsymbol{\lambda}^{\left[M_{\mathrm{I}}, M_{\mathrm{II}}\right]} \stackrel{\text { def }}{=} \boldsymbol{\lambda}+M_{\mathrm{I}} \tilde{\boldsymbol{\delta}}^{\mathrm{I}}+M_{\mathrm{II}} \tilde{\boldsymbol{\delta}}^{\mathrm{II}}
$$

The shift $\boldsymbol{\delta}$ and twisted shifts $\tilde{\boldsymbol{\delta}}^{\mathrm{I}}, \tilde{\boldsymbol{\delta}}^{\mathrm{II}}$ are given in Appendix for each polynomial. The denominator polynomial $\Xi_{\mathcal{D}}(x ; \boldsymbol{\lambda}) \stackrel{\text { def }}{=} \Xi_{\mathcal{D}}(\eta(x) ; \boldsymbol{\lambda})$ and the multi-indexed orthogonal polynomial $\check{P}_{\mathcal{D}, n}(x ; \boldsymbol{\lambda}) \stackrel{\text { def }}{=} P_{\mathcal{D}, n}(\eta(x) ; \boldsymbol{\lambda})$ are polynomials in $\eta(x)$ and their degrees are generically 1 $\ell_{\mathcal{D}}+n$, respectively, in which $\ell_{\mathcal{D}}$ is given by

$$
\begin{aligned}
\ell_{\mathcal{D}} & \stackrel{\text { def }}{=} \sum_{j=1}^{M_{\mathrm{I}}} d_{j}^{\mathrm{I}}-\frac{1}{2} M_{\mathrm{I}}\left(M_{\mathrm{I}}-1\right)+\sum_{j=1}^{M_{\mathrm{II}}} d_{j}^{\mathrm{II}}-\frac{1}{2} M_{\mathrm{II}}\left(M_{\mathrm{II}}-1\right)+M_{\mathrm{I}} M_{\mathrm{II}} \\
& =\sum_{j=1}^{M} d_{j}-\frac{1}{2} M(M-1)+2 M_{\mathrm{I}} M_{\mathrm{II}} .
\end{aligned}
$$

They are the polynomial parts of the Wronskian/Casoratian appearing in the eigenfunctions (2.9) $-(2.10)$ :

$$
\begin{aligned}
\check{\Xi}_{\mathcal{D}}(x ; \boldsymbol{\lambda}) & \propto \text { polynomial part of } \mathrm{W}_{\gamma}\left[\tilde{\phi}_{d_{1}^{\mathrm{I}}}^{\mathrm{I}}, \ldots, \tilde{\phi}_{d_{M_{\mathrm{I}}}^{\mathrm{I}}}^{\mathrm{I}}, \tilde{\phi}_{d_{1}^{\mathrm{II}}}^{\mathrm{II}}, \ldots, \tilde{\phi}_{d_{M_{\mathrm{II}}}^{\mathrm{II}}}^{\mathrm{II}}\right](x ; \boldsymbol{\lambda}), \\
\check{P}_{\mathcal{D}, n}(x ; \boldsymbol{\lambda}) & \propto \text { polynomial part of } \mathrm{W}_{\gamma}\left[\tilde{\phi}_{d_{1}^{\mathrm{I}}}^{\mathrm{I}}, \ldots, \tilde{\phi}_{d_{M_{\mathrm{I}}}^{\mathrm{I}}}^{\mathrm{I}}, \tilde{\phi}_{d_{1}^{\mathrm{II}}}^{\mathrm{II}}, \ldots, \tilde{\phi}_{d_{M_{\mathrm{II}}}^{\mathrm{II}}}^{\mathrm{II}}, \phi_{n}\right](x ; \boldsymbol{\lambda}),
\end{aligned}
$$

for $\mathrm{W}$ and $\mathrm{AW}$ cases $\left(\mathrm{W}_{\gamma}[\cdots]\right.$ are replaced by $\mathrm{W}[\cdots]$ for $\mathrm{L}$ and $\mathrm{J}$ cases $)$. We remark that the deformed Hamiltonian $\mathcal{H}_{\mathcal{D}}(\boldsymbol{\lambda})$ is independent of the order of $d_{j}$ but the denominator polynomial $\Xi_{\mathcal{D}}(\eta ; \boldsymbol{\lambda})$ and the multi-indexed orthogonal polynomial $P_{\mathcal{D}, n}(\eta ; \boldsymbol{\lambda})$ may change sign under the permutation of $d_{j}$ 's (The set $\mathcal{D}$ of $\Xi_{\mathcal{D}}(\eta ; \boldsymbol{\lambda})$ and $P_{\mathcal{D}, n}(\eta ; \boldsymbol{\lambda})$ is understood as an ordered set). The explicit forms of $\Xi_{\mathcal{D}}(\eta ; \boldsymbol{\lambda})$ and $P_{\mathcal{D}, n}(\eta ; \boldsymbol{\lambda})$ are given in Appendix.

The deformed Hamiltonian $\mathcal{H}_{\mathcal{D}}(\boldsymbol{\lambda})$ is non-singular for large enough $g(\mathrm{~L})$, large enough $g$ and $h(\mathrm{~J})$, large enough $\operatorname{Re} a_{j}(\forall j)(\mathrm{W})$ and small enough $\left|a_{j}\right|(\forall j)(\mathrm{AW})$. For more details, see [5, 27].

\section{Pseudo Virtual States and Wronskian/Casoratian Identities}

By using pseudo virtual states which are obtained from eigenstate by using the discrete symmetry of the original Hamiltonian, some Wronskian identities [18] and Casoratian identities [30] are derived. These identities imply the equivalence of two deformed quantum systems

\footnotetext{
${ }^{1}$ For specific values of parameters, the coefficient of the highest power term may vanish, see $\S$ A.4.3.
} 
obtained by Darboux-Crum transformation in terms of pseudo virtual state wavefunctions and eigenfunctions with shifted parameters. Here we extend these results. The data of pseudo virtual states are given in Appendix.

First we consider $\mathrm{W}$ and $\mathrm{AW}$ cases. In [30] the followings are shown. Let a set $\mathcal{D}$ and an integer $N$ be

$$
\mathcal{D}=\left\{d_{1}, \ldots, d_{M}\right\} \quad\left(d_{j} \in \mathbb{Z}_{\geq 0}, \text { mutually distinct }\right), \quad N \geq \max \left\{d_{1}, \ldots, d_{M}\right\}
$$

A set $\overline{\mathcal{D}}$ and $\overline{\boldsymbol{\lambda}}$ are defined by

$$
\begin{aligned}
& \overline{\mathcal{D}} \stackrel{\text { def }}{=}\{0,1, \ldots, N\} \backslash\left\{N-d_{1}, \ldots, N-d_{M}\right\} \stackrel{\text { def }}{=}\left\{e_{1}, \ldots, e_{N+1-M}\right\}, \\
& \overline{\boldsymbol{\lambda}} \stackrel{\text { def }}{=} \boldsymbol{\lambda}-(N+1) \boldsymbol{\delta} .
\end{aligned}
$$

Deform the original systems $\mathcal{H}(\boldsymbol{\lambda})$ and $\mathcal{H}(\overline{\boldsymbol{\lambda}})$ by Darboux-Crum transformation with the following seed solutions:

$$
\begin{aligned}
& \mathcal{H}(\boldsymbol{\lambda}) \text { with } \tilde{\phi}_{d_{1}}^{\mathrm{pvs}}(x ; \boldsymbol{\lambda}), \ldots, \tilde{\phi}_{d_{M}}^{\mathrm{pvs}}(x ; \boldsymbol{\lambda}) \Rightarrow \mathcal{H}_{\mathcal{D}}^{\mathrm{pvs}}(\boldsymbol{\lambda}) \\
& \mathcal{H}(\overline{\boldsymbol{\lambda}}) \text { with } \phi_{e_{1}}(x ; \overline{\boldsymbol{\lambda}}), \ldots, \phi_{e_{N+1} M}(x ; \overline{\boldsymbol{\lambda}}) \Rightarrow \mathcal{H}_{\overline{\mathcal{D}}}^{\mathrm{KA}}(\overline{\boldsymbol{\lambda}})
\end{aligned}
$$

The denominator polynomials $\Xi_{\mathcal{D}}^{\text {pvs }}(\eta ; \boldsymbol{\lambda})$ and $\bar{\Xi}_{\overline{\mathcal{D}}}(\eta ; \overline{\boldsymbol{\lambda}})$ are given by

$$
\begin{aligned}
\Xi_{\mathcal{D}}^{\mathrm{pvs}}(\eta(x) ; \boldsymbol{\lambda}) & \propto \text { polynomial part of } \mathrm{W}_{\gamma}\left[\tilde{\phi}_{d_{1}}^{\mathrm{pvs}}, \ldots, \tilde{\phi}_{d_{M}}^{\mathrm{pvs}}\right](x ; \boldsymbol{\lambda}), \\
\bar{\Xi}_{\overline{\mathcal{D}}}(\eta(x) ; \overline{\boldsymbol{\lambda}}) & \propto \text { polynomial part of } \mathrm{W}_{\gamma}\left[\phi_{e_{1}}, \ldots, \phi_{e_{N+1-M}}\right](x ; \overline{\boldsymbol{\lambda}}) .
\end{aligned}
$$

Then we have the Casoratian identity

$$
\Xi_{\mathcal{D}}^{\mathrm{pvs}}(\eta ; \boldsymbol{\lambda}) \propto \bar{\Xi}_{\overline{\mathcal{D}}}(\eta ; \overline{\boldsymbol{\lambda}})
$$

and this implies the equivalence of two deformed systems

$$
\mathcal{H}_{\mathcal{D}}^{\mathrm{pvs}}(\boldsymbol{\lambda})-\mathcal{E}_{-N-1}(\boldsymbol{\lambda})=\kappa^{-N-1} \mathcal{H}_{\overline{\mathcal{D}}}^{\mathrm{KA}}(\overline{\boldsymbol{\lambda}})
$$

These Hamiltonians are non-singular if the index set $\overline{\mathcal{D}}$ satisfies Krein-Adler (KA) condition, $\prod_{j=1}^{N+1-M}\left(n-e_{j}\right) \geq 0\left(\forall n \in \mathbb{Z}_{\geq 0}\right)[34,21]$. These relations are based on the energy relation

$$
\tilde{\mathcal{E}}_{\mathrm{v}}^{\mathrm{pvs}}(\boldsymbol{\lambda})-\mathcal{E}_{-N-1}(\boldsymbol{\lambda})=\kappa^{-N-1} \mathcal{E}_{N-\mathrm{v}}(\overline{\boldsymbol{\lambda}})
$$

The choice of the integer $N$ is not unique and the systems with different $N$ are related by shape-invariance. 
The spectrum of $\mathcal{H}_{\mathcal{D}}^{\mathrm{pvs}}(\boldsymbol{\lambda})$ and $\mathcal{H}_{\overline{\mathcal{D}}}^{\mathrm{KA}}(\overline{\boldsymbol{\lambda}})$ are

$$
\begin{array}{lll}
\mathcal{H}_{\mathcal{D}}^{\mathrm{pvs}}(\boldsymbol{\lambda}): & \tilde{\mathcal{E}}_{\mathrm{v}}^{\mathrm{pvs}}(\boldsymbol{\lambda})(\mathrm{v} \in \mathcal{D}), \quad \mathcal{E}_{n}(\boldsymbol{\lambda})\left(n \in \mathbb{Z}_{\geq 0}\right), \\
\mathcal{H}_{\overline{\mathcal{D}}}^{\mathrm{KA}}(\overline{\boldsymbol{\lambda}}): & \mathcal{E}_{N-\mathrm{v}}(\overline{\boldsymbol{\lambda}})(\mathrm{v} \in \mathcal{D}), & \mathcal{E}_{N+1+n}(\overline{\boldsymbol{\lambda}})\left(n \in \mathbb{Z}_{\geq 0}\right),
\end{array}
$$

and the energy eigenvalues satisfy

$$
\mathcal{E}_{n}(\boldsymbol{\lambda})-\mathcal{E}_{-N-1}(\boldsymbol{\lambda})=\kappa^{-N-1} \mathcal{E}_{N+1+n}(\overline{\boldsymbol{\lambda}})
$$

Therefore we can further perform Darboux-Crum transformation taking these eigenfunctions with energy $\mathcal{E}_{n}(\boldsymbol{\lambda})$ and $\mathcal{E}_{N+1+n}(\overline{\boldsymbol{\lambda}})$ as seed solutions, which originate from $\phi_{n}(x ; \boldsymbol{\lambda})$ and $\phi_{N+1+n}(x ; \overline{\boldsymbol{\lambda}})$, for $\mathcal{H}_{\mathcal{D}}^{\mathrm{pvs}}(\boldsymbol{\lambda})$ and $\mathcal{H}_{\overline{\mathcal{D}}}^{\mathrm{KA}}(\overline{\boldsymbol{\lambda}})$ respectively. Let the set of $M^{\prime}$ integers for this extra deletion be $\left\{d_{1}^{\prime}, \ldots, d_{M^{\prime}}^{\prime}\right\}\left(d_{j}^{\prime} \in \mathbb{Z}_{\geq 0}\right.$, mutually distinct). Then we have

$$
\begin{array}{llll}
\mathcal{H}_{\mathcal{D}}^{\mathrm{pvs}}(\boldsymbol{\lambda}) & \text { with } & \phi_{\mathcal{D} d_{1}^{\prime}}^{\mathrm{pvs}}(x ; \boldsymbol{\lambda}), \ldots, \phi_{\mathcal{D} d_{M^{\prime}}^{\prime}}^{\mathrm{pvs}}(x ; \boldsymbol{\lambda}) & \Rightarrow \mathcal{H}_{\mathcal{D}_{\mathrm{ext}}}^{\mathrm{pvs}+\mathrm{es}}(\boldsymbol{\lambda}), \\
\mathcal{H}_{\overline{\mathcal{D}}}^{\mathrm{KA}}(\overline{\boldsymbol{\lambda}}) & \text { with } & \phi_{\overline{\mathcal{D}} N+1+d_{1}^{\prime}}^{\mathrm{KA}}(x ; \overline{\boldsymbol{\lambda}}), \ldots, \phi_{\overline{\mathcal{D}} N+1+d_{M^{\prime}}^{\prime}}^{\mathrm{KA}}(x ; \overline{\boldsymbol{\lambda}}) \Rightarrow \mathcal{H}_{\overline{\mathcal{D}} \text { ext }}^{\mathrm{KA}}(\overline{\boldsymbol{\lambda}}),
\end{array}
$$

namely

$\mathcal{H}(\boldsymbol{\lambda})$ with $\tilde{\phi}_{d_{1}}^{\mathrm{pvs}}(x ; \boldsymbol{\lambda}), \ldots, \tilde{\phi}_{d_{M}}^{\mathrm{pvs}}(x ; \boldsymbol{\lambda}), \phi_{d_{1}^{\prime}}(x ; \boldsymbol{\lambda}), \ldots, \phi_{d_{M^{\prime}}^{\prime}}(x ; \boldsymbol{\lambda}) \Rightarrow \mathcal{H}_{\mathcal{D}_{\mathrm{ext}}}^{\mathrm{pvs}+\mathrm{es}}(\boldsymbol{\lambda})$ $\mathcal{H}(\overline{\boldsymbol{\lambda}})$ with $\phi_{e_{1}}(x ; \overline{\boldsymbol{\lambda}}), \ldots, \phi_{e_{N+1-M}}(x ; \overline{\boldsymbol{\lambda}}), \phi_{N+1+d_{1}^{\prime}}(x ; \overline{\boldsymbol{\lambda}}), \ldots, \phi_{N+1+d_{M^{\prime}}^{\prime}}(x ; \overline{\boldsymbol{\lambda}}) \Rightarrow \mathcal{H}_{\overline{\mathcal{D}}_{\text {ext }}^{\mathrm{KA}}}^{\mathrm{A}}(\overline{\boldsymbol{\lambda}})$,

where

$$
\begin{aligned}
& \mathcal{D}_{\text {ext }}=\left\{d_{1}, \ldots, d_{M}, d_{1}^{\prime}, \ldots, d_{M^{\prime}}^{\prime}\right\} \\
& \overline{\mathcal{D}}_{\text {ext }}=\left\{e_{1}, \ldots, e_{N+1-M}, N+1+d_{1}^{\prime}, \ldots, N+1+d_{M^{\prime}}^{\prime}\right\} .
\end{aligned}
$$

Since the same procedure is applied to equivalent systems, we have

$$
\mathcal{H}_{\mathcal{D}_{\text {ext }}^{\mathrm{pvs}}}^{\mathrm{ees}}(\boldsymbol{\lambda})-\mathcal{E}_{-N-1}(\boldsymbol{\lambda})=\kappa^{-N-1} \mathcal{H}_{\overline{\mathcal{D}}_{\text {ext }}^{\mathrm{KA}}}^{\mathrm{KA}}(\overline{\boldsymbol{\lambda}})
$$

These Hamiltonians are non-singular if $\overline{\mathcal{D}}_{\text {ext }}$ satisfies KA condition. By defining $\Xi_{\mathcal{D}_{\text {ext }}^{\text {pvs }}}^{\text {Kes }}(\eta ; \boldsymbol{\lambda})$ and $\bar{\Xi}_{\overline{\mathcal{D}}_{\text {ext }}}(\eta ; \overline{\boldsymbol{\lambda}})$ as

$$
\begin{aligned}
& \Xi_{\mathcal{D}_{\text {ext }}}^{\text {pvs+es }}(\eta(x) ; \boldsymbol{\lambda}) \propto \text { polynomial part of } \mathrm{W}_{\gamma}\left[\tilde{\phi}_{d_{1}}^{\mathrm{pvs}}, \ldots, \tilde{\phi}_{d_{M}}^{\mathrm{pvs}}, \phi_{d_{1}^{\prime}}, \ldots, \phi_{d_{M^{\prime}}^{\prime}}\right](x ; \boldsymbol{\lambda}), \\
& \bar{\Xi}_{\overline{\mathcal{D}}_{\text {ext }}}(\eta(x) ; \overline{\boldsymbol{\lambda}}) \propto \text { polynomial part of } \\
& \qquad \mathrm{W}_{\gamma}\left[\phi_{e_{1}}, \ldots, \phi_{e_{N+1-M}}, \phi_{N+1+d_{1}^{\prime}}, \ldots, \phi_{N+1+d_{M^{\prime}}^{\prime}}\right](x ; \overline{\boldsymbol{\lambda}}),
\end{aligned}
$$


we obtain

$$
\Xi_{\mathcal{D}_{\text {ext }}}^{\text {pvs+es }}(\eta ; \boldsymbol{\lambda}) \propto \bar{\Xi}_{\overline{\mathcal{D}}_{\text {ext }}}(\eta ; \overline{\boldsymbol{\lambda}})
$$

This polynomial proportionality (3.15) and equality (3.12) as an algebraic equation are valid for any parameter ranges (except for the specific values mentioned in footnote 1).

For $\mathrm{L}$ and $\mathrm{J}$ cases, the above argument is valid by replacing Casoratian $\mathrm{W}_{\gamma}[\cdots]$ with Wronskian W[*] and setting $\kappa=1$. Eqs.(3.6) and (3.7) are shown in [18]. Further DarbouxCrum transformation in terms of eigenfunctions gives (3.12) and (3.15).

\section{Equivalence of Multi-Indexed Orthogonal Polynomi- als}

We will show that the deformed system obtained by the Darboux-Crum transformations in terms of both type I and II virtual state wavefunctions is equivalent to that of type I only (or type II only) with shifted parameters. This means that multi-indexed orthogonal polynomials with both type I and II indices are proportional to that of type I only (or type II only) with shifted parameters. Our starting point is the proportionality of denominator

polynomials (3.15), namely (changing the notation : $\left(d_{j}, d_{j}^{\prime}, M, M^{\prime}\right) \rightarrow\left(d_{j}^{\prime}, d_{j}^{\prime \prime}, M^{\prime}, M^{\prime \prime}\right)$. $\left.N \geq \max \left\{d_{1}^{\prime}, \ldots, d_{M^{\prime}}^{\prime}\right\}\right)$,

polynomial part of $\mathrm{W}_{\gamma}\left[\tilde{\phi}_{d_{1}^{\prime}}^{\mathrm{pvs}}, \ldots, \tilde{\phi}_{d_{M^{\prime}}^{\prime}}^{\mathrm{pvs}}, \phi_{d_{1}^{\prime \prime}}, \ldots, \phi_{d_{M^{\prime \prime}}^{\prime \prime}}\right](x ; \boldsymbol{\lambda})$

$\propto$ polynomial part of $\mathrm{W}_{\gamma}\left[\phi_{e_{1}}, \ldots, \phi_{e_{N+1-M^{\prime}}}, \phi_{N+1+d_{1}^{\prime \prime}}, \ldots, \phi_{N+1+d_{M^{\prime \prime}}^{\prime \prime}}\right](x ; \boldsymbol{\lambda}-(N+1) \boldsymbol{\delta})$,

or its Wronskian version. This relation is valid for any parameter ranges (except for specific parameter values mentioned in footnote 1). Various data are given in Appendix.

\subsection{Wilson and Askey-Wilson}

First we consider W and AW cases. From the relation between (pseudo) virtual state and eigenstate

$$
\tilde{\phi}_{\mathrm{v}}^{\mathrm{I}}(x ; \boldsymbol{\lambda})=\phi_{\mathrm{v}}\left(x ; \mathfrak{t}^{\mathrm{I}}(\boldsymbol{\lambda})\right), \quad \tilde{\phi}_{\mathrm{v}}^{\mathrm{II}}(x ; \boldsymbol{\lambda})=\phi_{\mathrm{v}}\left(x ; \mathfrak{t}^{\mathrm{II}}(\boldsymbol{\lambda})\right), \quad \tilde{\phi}_{\mathrm{v}}^{\mathrm{pvs}}(x ; \boldsymbol{\lambda})=\phi_{\mathrm{v}}(x ; \mathfrak{t}(\boldsymbol{\lambda})),
$$

and $\mathfrak{t}=\mathfrak{t}^{\mathrm{II}} \circ \mathfrak{t}^{\mathrm{I}}$, we have

$$
\tilde{\phi}_{\mathrm{v}}^{\mathrm{pvs}}\left(x ; \mathfrak{t}^{\mathrm{I}}(\boldsymbol{\lambda})\right)=\tilde{\phi}_{\mathrm{v}}^{\mathrm{II}}(x ; \boldsymbol{\lambda}), \quad \tilde{\phi}_{\mathrm{v}}^{\mathrm{pvs}}\left(x ; \mathfrak{t}^{\mathrm{II}}(\boldsymbol{\lambda})\right)=\tilde{\phi}_{\mathrm{v}}^{\mathrm{I}}(x ; \boldsymbol{\lambda}) .
$$


For the index set $\mathcal{D}$ (2.11) , we take an integer $N$ and define an index set $\overline{\mathcal{D}}_{\mathrm{I}}$

$$
\begin{aligned}
N & \geq d_{\mathrm{II}}^{\max } \stackrel{\stackrel{\text { def }}{=}}{\max }\left\{d_{1}^{\mathrm{II}}, \ldots, d_{M_{\mathrm{II}}}^{\mathrm{II}}\right\} \quad(\max \{\cdot\} \stackrel{\text { def }}{=}-1), \\
\overline{\mathcal{D}}_{\mathrm{I}} & \stackrel{\text { def }}{=}\left(\{0,1, \ldots, N\} \backslash\left\{N-d_{1}^{\mathrm{II}}, \ldots, N-d_{M_{\mathrm{II}}}^{\mathrm{II}}\right\}\right) \cup\left\{N+1+d_{1}^{\mathrm{I}}, \ldots, N+1+d_{M_{\mathrm{I}}}^{\mathrm{I}}\right\} \\
& \stackrel{\text { def }}{=}\left\{e_{1}^{\mathrm{I}}, e_{2}^{\mathrm{I}}, \ldots, e_{N+1-M_{\mathrm{II}}+M_{\mathrm{I}}}^{\mathrm{I}}\right\} .
\end{aligned}
$$

Since (4.1) holds for any parameter ranges, let us substitute $\boldsymbol{\lambda} \rightarrow \mathfrak{t}^{\mathrm{I}}(\boldsymbol{\lambda})$ in (4.1). Then we have

$$
\begin{gathered}
\text { polynomial part of } \mathrm{W}_{\gamma}\left[\tilde{\phi}_{d_{1}^{\mathrm{II}}}^{\mathrm{II}}, \ldots, \tilde{\phi}_{d_{M_{\mathrm{II}}}^{\mathrm{II}}}^{\mathrm{II}}, \tilde{\phi}_{d_{1}^{\mathrm{I}}}^{\mathrm{I}}, \ldots, \tilde{\phi}_{d_{M_{\mathrm{I}}}^{\mathrm{I}}}^{\mathrm{I}}\right](x ; \boldsymbol{\lambda}) \\
\propto \text { polynomial part of } \mathrm{W}_{\gamma}\left[\tilde{\phi}_{e_{1}^{\mathrm{I}}}^{\mathrm{I}}, \ldots, \tilde{\phi}_{e_{N+1-M_{\mathrm{II}}+M_{\mathrm{I}}}^{\mathrm{I}}}^{\mathrm{I}}\right]\left(x ; \boldsymbol{\lambda}-(N+1) \tilde{\boldsymbol{\delta}}^{\mathrm{I}}\right),
\end{gathered}
$$

where $\mathfrak{t}^{\mathrm{I}}+\beta \boldsymbol{\delta}=\mathfrak{t}^{\mathrm{I}}\left(\boldsymbol{\lambda}+\beta \tilde{\boldsymbol{\delta}}^{\mathrm{I}}\right)$ is used. Therefore the denominator polynomial with both type I and II indices is proportional to one of type I only,

$$
\Xi_{\mathcal{D}}(\eta ; \boldsymbol{\lambda}) \propto \Xi_{\overline{\mathcal{D}}_{\mathrm{I}}}\left(\eta ; \boldsymbol{\lambda}-(N+1) \tilde{\boldsymbol{\delta}}^{\mathrm{I}}\right) .
$$

The relation $\tilde{\boldsymbol{\delta}}^{\mathrm{II}}=-\tilde{\boldsymbol{\delta}}^{\mathrm{I}}$ implies

$$
\boldsymbol{\lambda}-(N+1) \tilde{\boldsymbol{\delta}}^{\mathrm{I}}+\left(N+1-M_{\mathrm{II}}+M_{\mathrm{I}}\right) \tilde{\boldsymbol{\delta}}^{\mathrm{I}}=\boldsymbol{\lambda}^{\left[M_{\mathrm{I}}, M_{\mathrm{II}}\right]} .
$$

By using this, (4.6) and the general formula for potential function (2.15), we can show that

$$
V_{\mathcal{D}}(x ; \boldsymbol{\lambda})=V_{\overline{\mathcal{D}}_{\mathrm{I}}}\left(x ; \boldsymbol{\lambda}-(N+1) \tilde{\boldsymbol{\delta}}^{\mathrm{I}}\right) .
$$

Namely we obtain the equivalence of two deformed systems, type I and II mixed system and type I system with shifted parameter,

$$
\mathcal{H}_{\mathcal{D}}(\boldsymbol{\lambda})=\mathcal{H}_{\overline{\mathcal{D}}_{\mathrm{I}}}\left(\boldsymbol{\lambda}-(N+1) \tilde{\boldsymbol{\delta}}^{\mathrm{I}}\right) .
$$

This equality as an algebraic equation holds for any parameter ranges but this deformed Hamiltonian is non-singular only for restricted parameter ranges (see the end of $\S 2$ ). From the general formula (2.17), we can show

$$
\psi_{\mathcal{D}}(x ; \boldsymbol{\lambda}) \propto \psi_{\overline{\mathcal{D}}_{\mathrm{I}}}\left(x ; \boldsymbol{\lambda}-(N+1) \tilde{\boldsymbol{\delta}}^{\mathrm{I}}\right) .
$$

Therefore the general formula for eigenfunctions (2.16) gives

$$
P_{\mathcal{D}, n}(\eta ; \boldsymbol{\lambda}) \propto P_{\overline{\mathcal{D}}_{\mathrm{I}}, n}\left(\eta ; \boldsymbol{\lambda}-(N+1) \tilde{\boldsymbol{\delta}}^{\mathrm{I}}\right) \quad(n=0,1, \ldots) .
$$


This proportionality is valid for any parameter ranges.

Similarly, by substituting $\boldsymbol{\lambda} \rightarrow \mathfrak{t}^{\mathrm{II}}(\boldsymbol{\lambda})$ in (4.1), we can show that a type I and II mixed system is equivalent to a type II system with shifted parameters. Results are the following:

$$
\begin{aligned}
& N \geq d_{\mathrm{I}}^{\max } \stackrel{\text { def }}{=} \max \left\{d_{1}^{\mathrm{I}}, \ldots, d_{M_{\mathrm{I}}}^{\mathrm{I}}\right\} \\
& \overline{\mathcal{D}}_{\mathrm{II}} \stackrel{\text { def }}{=}\left(\{0,1, \ldots, N\} \backslash\left\{N-d_{1}^{\mathrm{I}}, \ldots, N-d_{M_{\mathrm{I}}}^{\mathrm{I}}\right\}\right) \cup\left\{N+1+d_{1}^{\mathrm{II}}, \ldots, N+1+d_{M_{\mathrm{II}}}^{\mathrm{II}}\right\} \\
& \quad \stackrel{\text { def }}{=}\left\{e_{1}^{\mathrm{II}}, e_{2}^{\mathrm{II}}, \ldots, e_{N+1-M_{\mathrm{I}}+M_{\mathrm{II}}}^{\mathrm{II}}\right\} \\
& \Xi_{\mathcal{D}}(\eta ; \boldsymbol{\lambda}) \propto \Xi_{\overline{\mathcal{D}}_{\mathrm{II}}}\left(\eta ; \boldsymbol{\lambda}-(N+1) \tilde{\boldsymbol{\delta}}^{\mathrm{II}}\right) \\
& \mathcal{H}_{\mathcal{D}}(\boldsymbol{\lambda})=\mathcal{H}_{\overline{\mathcal{D}}_{\mathrm{II}}}\left(\boldsymbol{\lambda}-(N+1) \tilde{\boldsymbol{\delta}}^{\mathrm{II}}\right) \\
& P_{\mathcal{D}, n}(\eta ; \boldsymbol{\lambda}) \propto P_{\overline{\mathcal{D}}_{\mathrm{II}}, n}\left(\eta ; \boldsymbol{\lambda}-(N+1) \tilde{\boldsymbol{\delta}}^{\mathrm{II}}\right) \quad(n=0,1, \ldots)
\end{aligned}
$$

We note that $\ell_{\mathcal{D}}=\ell_{\overline{\mathcal{D}}_{\mathrm{I}}}=\ell_{\overline{\mathcal{D}}_{\mathrm{II}}}$ and the energy eigenvalues are invariant under twisting shifts, $\mathcal{E}_{n}(\boldsymbol{\lambda})=\mathcal{E}_{n}\left(\boldsymbol{\lambda}+\tilde{\boldsymbol{\delta}}^{\mathrm{I}}\right)=\mathcal{E}_{n}\left(\boldsymbol{\lambda}+\tilde{\boldsymbol{\delta}}^{\mathrm{II}}\right)$ and

$$
\mathcal{E}_{n}(\boldsymbol{\lambda})=\mathcal{E}_{n}\left(\boldsymbol{\lambda}-(N+1) \tilde{\boldsymbol{\delta}}^{\mathrm{I}}\right)=\mathcal{E}_{n}\left(\boldsymbol{\lambda}-(N+1) \tilde{\boldsymbol{\delta}}^{\mathrm{II}}\right) .
$$

\section{$4.2 \quad$ Jacobi}

Next we consider Jacobi case. The argument presented in the previous subsection applies also to this case. From (4.1) we obtain the proportionality (4.6). By using (4.7), (4.6) and general formulas (2.13) and (2.16)-(2.18), we can show (4.10) and

$$
\phi_{\mathcal{D} 0}(x ; \boldsymbol{\lambda}) \propto \phi_{\overline{\mathcal{D}}_{\mathrm{I}} 0}\left(x ; \boldsymbol{\lambda}-(N+1) \tilde{\boldsymbol{\delta}}^{\mathrm{I}}\right), \quad U_{\mathcal{D}}(x ; \boldsymbol{\lambda})=U_{\overline{\mathcal{D}}_{\mathrm{I}}}\left(x ; \boldsymbol{\lambda}-(N+1) \tilde{\boldsymbol{\delta}}^{\mathrm{I}}\right)
$$

Therefore we obtain (4.9) and (4.11). We have also (4.12)-(4.17).

\subsection{Laguerre}

Lastly we consider Laguerre case. The (pseudo)virtual states are (see Appendix)

$$
\tilde{\phi}_{\mathrm{v}}^{\mathrm{I}}(x ; \boldsymbol{\lambda})=i^{-g} \phi_{\mathrm{v}}(i x ; \boldsymbol{\lambda}), \quad \tilde{\phi}_{\mathrm{v}}^{\mathrm{II}}(x ; \boldsymbol{\lambda})=\phi_{\mathrm{v}}(x ; \mathfrak{t}(\boldsymbol{\lambda})), \quad \tilde{\phi}_{\mathrm{v}}^{\mathrm{pvs}}(x ; \boldsymbol{\lambda})=i^{g-1} \phi_{\mathrm{v}}(i x ; \mathfrak{t}(\boldsymbol{\lambda})),
$$

and we have

$$
\tilde{\phi}_{\mathrm{v}}^{\mathrm{pvs}}(i x ; \boldsymbol{\lambda}) \propto \tilde{\phi}_{\mathrm{v}}^{\mathrm{II}}(x ; \boldsymbol{\lambda}), \quad \tilde{\phi}_{\mathrm{v}}^{\mathrm{pvs}}(x ; \mathfrak{t}(\boldsymbol{\lambda})) \propto \tilde{\phi}_{\mathrm{v}}^{\mathrm{I}}(x ; \boldsymbol{\lambda}) .
$$

The property of the Wronskian $\left(f_{j}=f_{j}(x)\right)$

$$
\mathrm{W}\left[f_{1}, f_{2}, \ldots, f_{n}\right](g(x))=\left(\frac{d g(x)}{d x}\right)^{-\frac{1}{2} n(n-1)} \mathrm{W}\left[F_{1}, F_{2}, \ldots, F_{n}\right](x), \quad F_{j}(x) \stackrel{\text { def }}{=} f_{j}(g(x)),
$$


gives $\mathrm{W}\left[f_{1}, \ldots, f_{n}\right](i x) \propto \mathrm{W}\left[f_{1}(i x), \ldots, f_{n}(i x)\right](x)$. By using these, (4.1) with the replacement $x \rightarrow i x$ leads to (4.6), and (4.1) with the replacement $\boldsymbol{\lambda} \rightarrow \mathfrak{t}(\boldsymbol{\lambda})$ leads to (4.14).

Once (4.6) and (4.14) are obtained, the remaining task is the same as Jacobi case. By using (4.7), (4.6) and general formulas (2.13) and (2.16)-(2.18), we can show (4.10) and (4.18). Therefore we obtain (4.9) and (4.11). We have also (4.12)-(4.17).

\subsection{Zero level deletion}

If the degree of virtual state wavefunction for deletion is zero $\left(d_{j}=0\right)$, an $M$-indexed orthogonal polynomial reduces to an $(M-1)$-indexed orthogonal polynomial [5, 27]:

$$
\begin{aligned}
\left.P_{\mathcal{D}, n}(\eta ; \boldsymbol{\lambda})\right|_{d_{M_{\mathrm{I}}}^{\mathrm{I}}=0} \propto & P_{\mathcal{D}^{\prime}, n}\left(x ; \boldsymbol{\lambda}+\tilde{\boldsymbol{\delta}}^{\mathrm{I}}\right), \\
& \mathcal{D}^{\prime}=\left\{d_{1}^{\mathrm{I}}-1, \ldots, d_{M_{\mathrm{I}}-1}^{\mathrm{I}}-1, d_{1}^{\mathrm{II}}+1, \ldots, d_{M_{\mathrm{II}}}^{\mathrm{II}}+1\right\} \\
\left.P_{\mathcal{D}, n}(x ; \boldsymbol{\lambda})\right|_{d_{M_{\mathrm{II}}}^{\mathrm{II}}=0} \propto & P_{\mathcal{D}^{\prime}, n}\left(x ; \boldsymbol{\lambda}+\tilde{\boldsymbol{\delta}}^{\mathrm{II}}\right), \\
& \mathcal{D}^{\prime}=\left\{d_{1}^{\mathrm{I}}+1, \ldots, d_{M_{\mathrm{I}}}^{\mathrm{I}}+1, d_{1}^{\mathrm{II}}-1, \ldots, d_{M_{\mathrm{II}}-1}^{\mathrm{II}}-1\right\}
\end{aligned}
$$

which are the consequences of shape-invariance. They can be rederived by (4.11) and (4.16). By using (4.11), the following index sets give equivalent multi-indexed orthogonal polynomials:

$$
\begin{aligned}
& \left\{d_{1}^{\mathrm{I}}, \ldots, d_{M_{\mathrm{I}}-1}^{\mathrm{I}}, 0, d_{1}^{\mathrm{II}}, \ldots, d_{\left.d_{M_{\mathrm{II}}}^{\mathrm{II}}\right\}}^{\mathrm{II}} \text { with } \boldsymbol{\lambda} \quad\left(N \geq \max \left\{d_{1}^{\mathrm{II}}, \ldots, d_{M_{\mathrm{II}}}^{\mathrm{II}}\right\}\right)\right. \\
\leftrightarrow & \left(\{0, \ldots, N\} \backslash\left\{N-d_{1}^{\mathrm{II}}, \ldots, N-d_{M_{\mathrm{II}}}^{\mathrm{II}}\right\}\right) \cup\left\{N+1+d_{1}^{\mathrm{I}}, \ldots, N+1+d_{M_{\mathrm{I}}-1}^{\mathrm{I}}, N+1\right\} \\
& (\text { all type I }) \quad \text { with } \boldsymbol{\lambda}-(N+1) \tilde{\boldsymbol{\delta}}^{\mathrm{I}} \\
= & \left(\{0, \ldots, N+1\} \backslash\left\{(N+1)-\left(d_{1}^{\mathrm{II}}+1\right), \ldots,(N+1)-\left(d_{M_{\mathrm{II}}}^{\mathrm{II}}+1\right)\right\}\right) \\
& \cup\left\{(N+1)+1+\left(d_{1}^{\mathrm{I}}-1\right), \ldots,(N+1)+1+\left(d_{M_{\mathrm{I}}-1}^{\mathrm{I}}-1\right)\right\} \quad(\text { all type I }) \\
& \quad \text { with } \boldsymbol{\lambda}+\tilde{\boldsymbol{\delta}}^{\mathrm{I}}-((N+1)+1) \tilde{\boldsymbol{\delta}}^{\mathrm{I}}\left(N+1 \geq \max \left\{d_{1}^{\mathrm{II}}+1, \ldots, d_{M_{\mathrm{II}}}^{\mathrm{II}}+1\right\}\right) \\
\leftrightarrow & \left\{d_{1}^{\mathrm{I}}-1, \ldots, d_{M_{\mathrm{I}}-1}^{\mathrm{I}}-1, d_{1}^{\mathrm{II}}+1, \ldots, d_{d_{M_{\mathrm{II}}}^{\mathrm{II}}}^{\mathrm{II}}+1\right\} \text { with } \boldsymbol{\lambda}+\tilde{\boldsymbol{\delta}}^{\mathrm{I}},
\end{aligned}
$$

which means (4.19). Similarly (4.20) is derived by (4.16).

\section{Summary and Comments}

Multi-indexed orthogonal polynomials of L, J, W and AW types are labeled by an index set $\mathcal{D}$ but different index sets may give the same multi-indexed orthogonal polynomials, 
$P_{\mathcal{D}, n}(\eta ; \boldsymbol{\lambda}) \propto P_{\mathcal{D}^{\prime}, n}\left(\eta ; \boldsymbol{\lambda}^{\prime}\right)$. Based on extensions of Wronskian/Casoratian identities obtained in [18, 30], we have shown equivalences of two deformed systems, (4.9) and (4.15). One system has both type I and type II indices and the other has type I indices only (or type II indices only). These equivalences of two systems imply the proportionalities of multi-indexed orthogonal polynomials, (4.11) and (4.16). Redundant $N$-dependence in (4.11) and (4.16) comes from shape-invariance. Two deformed systems with index sets of the form (2.11) (with $d_{j}>0$ ) are equivalent, when the corresponding two $\overline{\mathcal{D}}_{\mathrm{I}}$ (4.4) (or $\overline{\mathcal{D}}_{\text {II }}$ (4.13)) with the minimum $N$ are equal as a set (and parameters $\boldsymbol{\lambda}$ are appropriately shifted).

For the index sets with type I indices only (or type II indices only), are there equivalences among them? We conjecture that there are no equivalences among them (for generic parameters). There are accidental coincidences of the denominator polynomials for low degree cases, for example,

$$
\begin{aligned}
& \mathcal{D}=\{2\}, \quad \mathcal{D}^{\prime}=\{1,2\}, \quad \Xi_{\mathcal{D}}(\eta ; \boldsymbol{\lambda}) \propto \Xi_{\mathcal{D}^{\prime}}\left(\eta ; \boldsymbol{\lambda}^{\prime}\right), \\
& \text { type I only : } \quad \boldsymbol{\lambda}^{\prime}=\left\{\begin{array}{ll}
(-2-g, 4-h) & : \mathrm{J} \\
\left(\frac{5}{2}-\lambda_{1}, \frac{5}{2}-\lambda_{2},-\frac{1}{2}-\lambda_{3},-\frac{1}{2}-\lambda_{4}\right) & : \mathrm{W}, \mathrm{AW}
\end{array},\right. \\
& \text { type II only : } \quad \boldsymbol{\lambda}^{\prime}= \begin{cases}(4-g,-2-h) & : \mathrm{J} \\
\left(-\frac{1}{2}-\lambda_{1},-\frac{1}{2}-\lambda_{2}, \frac{5}{2}-\lambda_{3}, \frac{5}{2}-\lambda_{4}\right) & : \mathrm{W}, \mathrm{AW}\end{cases}
\end{aligned}
$$

However these proportionalities of the denominator polynomials do not mean those of the multi-indexed orthogonal polynomials. In fact, we have $P_{\mathcal{D}, n}(\eta ; \boldsymbol{\lambda}) \propto P_{\mathcal{D}^{\prime}, n}\left(\eta ; \boldsymbol{\lambda}^{\prime}-2 \boldsymbol{\delta}\right)$ for $n=0,1$ but $P_{\mathcal{D}, n}(\eta ; \boldsymbol{\lambda}) \not \subset P_{\mathcal{D}^{\prime}, n}\left(\eta ; \boldsymbol{\lambda}^{\prime}-2 \boldsymbol{\delta}\right)$ for $n \geq 2$.

Corresponding to the three term recurrence relations for ordinary orthogonal polynomials, $M$-indexed orthogonal polynomials satisfy $3+2 M$ term recurrence relations [28]. When we calculate multi-indexed orthogonal polynomials explicitly, this $3+2 M$ term recurrence relation provides us more effective calculation method compared to their original definitions which are expressed in terms of determinant. If the $M$-indexed orthogonal polynomials $P_{\mathcal{D}, n}(\eta ; \boldsymbol{\lambda})$ are equivalent to $M^{\prime}$-indexed one $P_{\mathcal{D}^{\prime}, n}\left(\eta ; \boldsymbol{\lambda}^{\prime}\right)\left(M^{\prime}<M\right)$, these $P_{\mathcal{D}, n}(\eta ; \boldsymbol{\lambda})$ also satisfy $3+2 M^{\prime}$ term recurrence relations. For an index set $\mathcal{D}(2.11)$ with parameters $\boldsymbol{\lambda}$, this happens in the following cases (0)-(iv):

$$
\text { (0) } d_{j}=0: \mathcal{D}^{\prime} \text { is given by (4.19) or (4.20) }\left(M^{\prime}=M-1\right) \text {. }
$$

In the following we assume $d_{j}>0$ and set $I_{1} \stackrel{\text { def }}{=} 2 M_{\mathrm{I}}-d_{\mathrm{I}}^{\max }-1$ and $I_{2} \stackrel{\text { def }}{=} 2 M_{\mathrm{II}}-d_{\mathrm{II}}^{\max }-1$. We present $\mathcal{D}^{\prime}$ with the minimum $M^{\prime}$. For $M_{\mathrm{I}}, M_{\text {II }}>0$ cases, we have

(i) $I_{1} \leq 0<I_{2}$ or $0<I_{1} \leq I_{2}: \mathcal{D}^{\prime}=\left.\overline{\mathcal{D}}_{\mathrm{I}}\right|_{N=d_{\mathrm{II}}^{\max }}$ with $\boldsymbol{\lambda}-\left(d_{\mathrm{II}}^{\max }+1\right) \tilde{\boldsymbol{\delta}}^{\mathrm{I}}\left(M^{\prime}=M-I_{2}\right)$, 
(ii) $I_{2} \leq 0<I_{1}$ or $0<I_{2} \leq I_{1}: \mathcal{D}^{\prime}=\left.\overline{\mathcal{D}}_{\mathrm{II}}\right|_{N=d_{\mathrm{I}}^{\max }}$ with $\boldsymbol{\lambda}-\left(d_{\mathrm{I}}^{\max }+1\right) \tilde{\boldsymbol{\delta}}^{\mathrm{II}}\left(M^{\prime}=M-I_{1}\right)$.

For $M_{\mathrm{I}}=0$ or $M_{\mathrm{II}}=0$ cases, let us assume $d_{1}<\cdots<d_{M}$ and use the formulas in the opposite direction. Then we have for $M_{\mathrm{II}}=0$,

$$
\begin{aligned}
& \text { (iii) } \exists j \text { s.t. } 2 j-1-d_{j}>0 \text { : } \\
& \mathcal{D}^{\prime}=\left\{\left(d_{L+1}-N-1\right)^{\mathrm{I}}, \ldots,\left(d_{M}-N-1\right)^{\mathrm{I}}\right\} \cup\left\{d_{1}^{\mathrm{II}}, \ldots, d_{N+1-L}^{\mathrm{II}}\right\}, \quad N=d_{L}, \\
& \quad \text { with } \boldsymbol{\lambda}+\left(d_{L}+1\right) \tilde{\boldsymbol{\delta}}^{\mathrm{I}}\left(M^{\prime}=M-\left(2 L-1-d_{L}\right)\right),
\end{aligned}
$$

where $L$ is $j$ giving the maximum value of $2 j-1-d_{j}$ ( $L$ may not be unique) and $d_{i}^{\mathrm{II}}$ 's are determined by

$$
\{0,1, \ldots, N\} \backslash\left\{N-d_{1}^{\mathrm{II}}, \ldots, N-d_{N+1-L}^{\mathrm{II}}\right\}=\left\{d_{1}, \ldots, d_{L}\right\}
$$

The case $M_{\mathrm{I}}=0$ is similar,

(iv) $\exists j$ s.t. $2 j-1-d_{j}>0$ :

$$
\begin{aligned}
& \mathcal{D}^{\prime}=\left\{\left(d_{L+1}-N-1\right)^{\mathrm{II}}, \ldots,\left(d_{M}-N-1\right)^{\mathrm{II}}\right\} \cup\left\{d_{1}^{\mathrm{I}}, \ldots, d_{N+1-L}^{\mathrm{I}}\right\}, \quad N=d_{L}, \\
& \text { with } \boldsymbol{\lambda}+\left(d_{L}+1\right) \tilde{\boldsymbol{\delta}}^{\mathrm{II}}\left(M^{\prime}=M-\left(2 L-1-d_{L}\right)\right),
\end{aligned}
$$

where $L$ is same as (iii) and $d_{i}^{\mathrm{I}}$ 's are determined by $\{0,1, \ldots, N\} \backslash\left\{N-d_{1}^{\mathrm{I}}, \ldots, N-d_{N+1-L}^{\mathrm{I}}\right\}=$ $\left\{d_{1}, \ldots, d_{L}\right\}$. For illustration we present examples:

(i) : $\mathcal{D}=\left\{1^{\mathrm{I}}, 4^{\mathrm{I}}, 1^{\mathrm{II}}, 2^{\mathrm{II}}\right\}$ with $\boldsymbol{\lambda} \leftrightarrow \mathcal{D}^{\prime}=\left\{2^{\mathrm{I}}, 4^{\mathrm{I}}, 7^{\mathrm{I}}\right\}$ with $\boldsymbol{\lambda}-3 \tilde{\boldsymbol{\delta}}^{\mathrm{I}}$,

(ii) : $\mathcal{D}=\left\{1^{\mathrm{I}}, 2^{\mathrm{I}}, 4^{\mathrm{I}}, 2^{\mathrm{II}}, 3^{\mathrm{II}}\right\}$ with $\boldsymbol{\lambda} \leftrightarrow \mathcal{D}^{\prime}=\left\{1^{\mathrm{II}}, 4^{\mathrm{II}}, 7^{\mathrm{II}}, 8^{\mathrm{II}}\right\}$ with $\boldsymbol{\lambda}-5 \tilde{\boldsymbol{\delta}}^{\mathrm{II}}$,

(iii) : $\mathcal{D}=\left\{1^{\mathrm{I}}, 2^{\mathrm{I}}, 3^{\mathrm{I}}, 4^{\mathrm{I}}, 5^{\mathrm{I}}\right\}$ with $\boldsymbol{\lambda} \leftrightarrow \mathcal{D}^{\prime}=\left\{5^{\mathrm{II}}\right\}$ with $\boldsymbol{\lambda}+6 \tilde{\boldsymbol{\delta}}^{\mathrm{I}}$,

$$
\begin{aligned}
\mathcal{D}=\left\{2^{\mathrm{I}}, 3^{\mathrm{I}}, 4^{\mathrm{I}}, 7^{\mathrm{I}}\right\} \text { with } \boldsymbol{\lambda} & \leftrightarrow \mathcal{D}^{\prime}=\left\{2^{\mathrm{I}}, 3^{\mathrm{II}}, 4^{\mathrm{II}}\right\} \text { with } \boldsymbol{\lambda}+5 \tilde{\boldsymbol{\delta}}^{\mathrm{I}}, \\
\mathcal{D}=\left\{1^{\mathrm{I}}, 2^{\mathrm{I}}, 5^{\mathrm{I}}, 6^{\mathrm{I}}\right\} \text { with } \boldsymbol{\lambda} & \leftrightarrow \mathcal{D}^{\prime}=\left\{2^{\mathrm{II}}, 3^{\mathrm{II}}, 6^{\mathrm{II}}\right\} \text { with } \boldsymbol{\lambda}+7 \tilde{\boldsymbol{\delta}}^{\mathrm{I}} \\
& \leftrightarrow \mathcal{D}^{\prime}=\left\{2^{\mathrm{I}}, 3^{\mathrm{I}}, 2^{\mathrm{II}}\right\} \text { with } \boldsymbol{\lambda}+3 \tilde{\boldsymbol{\delta}}^{\mathrm{I}},
\end{aligned}
$$

(iv) : $\mathcal{D}=\left\{1^{\mathrm{II}}, 3^{\mathrm{II}}, 4^{\mathrm{II}}, 5^{\mathrm{II}}, 8^{\mathrm{II}}\right\}$ with $\boldsymbol{\lambda} \leftrightarrow \mathcal{D}^{\prime}=\left\{3^{\mathrm{I}}, 5^{\mathrm{I}}, 2^{\mathrm{II}}\right\}$ with $\boldsymbol{\lambda}+6 \tilde{\boldsymbol{\delta}}^{\mathrm{II}}$.

Note added: After submitting the manuscript (arXiv:1309.2346[math-ph]), we have noticed that the proportionalities (4.11) and (4.16) can be directly derived by repeated use of (4.19) and (4.20), which is an idea of K. Takemura (private communication, [36]). 


\section{Acknowledgements}

I thank R. Sasaki for useful discussion and reading of the manuscript, and K. Takemura for useful discussion. I am supported in part by Grant-in-Aid for Scientific Research from the Ministry of Education, Culture, Sports, Science and Technology (MEXT), No.25400395.

\section{A Various Data}

For reader's convenience, we present various data of quantum mechanical systems described by Laguerre(L), Jacobi(J), Wilson(W) and Askey-Wilson(AW) polynomials [5, 27, 18, 30].

\section{A.1 Original systems}

The Hamiltonian and eigenfunctions have the following form

$$
\begin{aligned}
& \mathcal{H}(\boldsymbol{\lambda})=\mathcal{A}(\boldsymbol{\lambda})^{\dagger} \mathcal{A}(\boldsymbol{\lambda}), \\
& \phi_{n}(x ; \boldsymbol{\lambda})=\phi_{0}(x ; \boldsymbol{\lambda}) \check{P}_{n}(x ; \boldsymbol{\lambda}), \quad \check{P}_{n}(x ; \boldsymbol{\lambda}) \stackrel{\text { def }}{=} P_{n}(\eta(x) ; \boldsymbol{\lambda}),
\end{aligned}
$$

and the systems are shape-invariant

$$
\mathcal{A}(\boldsymbol{\lambda}) \mathcal{A}(\boldsymbol{\lambda})^{\dagger}=\kappa \mathcal{A}(\boldsymbol{\lambda}+\boldsymbol{\delta})^{\dagger} \mathcal{A}(\boldsymbol{\lambda}+\boldsymbol{\delta})+\mathcal{E}_{1}(\boldsymbol{\lambda})
$$

which implies the forward and backward shift relations

$$
\begin{aligned}
& \mathcal{A}(\boldsymbol{\lambda}) \phi_{n}(x ; \boldsymbol{\lambda})=f_{n}(\boldsymbol{\lambda}) \phi_{n-1}(x ; \boldsymbol{\lambda}+\boldsymbol{\delta}), \quad \mathcal{A}(\boldsymbol{\lambda})^{\dagger} \phi_{n-1}(x ; \boldsymbol{\lambda}+\boldsymbol{\delta})=b_{n-1}(\boldsymbol{\lambda}) \phi_{n}(x ; \boldsymbol{\lambda}), \\
& \mathcal{F}(\boldsymbol{\lambda}) \check{P}_{n}(x ; \boldsymbol{\lambda})=f_{n}(\boldsymbol{\lambda}) \check{P}_{n-1}(x ; \boldsymbol{\lambda}+\boldsymbol{\delta}), \quad \mathcal{B}(\boldsymbol{\lambda}) \check{P}_{n-1}(x ; \boldsymbol{\lambda}+\boldsymbol{\delta})=b_{n-1}(\boldsymbol{\lambda}) \check{P}_{n}(x ; \boldsymbol{\lambda}), \\
& \mathcal{F}(\boldsymbol{\lambda}) \stackrel{\text { def }}{=} \phi_{0}(x ; \boldsymbol{\lambda}+\boldsymbol{\delta})^{-1} \circ \mathcal{A}(\boldsymbol{\lambda}) \circ \phi_{0}(x ; \boldsymbol{\lambda}), \mathcal{B}(\boldsymbol{\lambda}) \stackrel{\text { def }}{=} \phi_{0}(x ; \boldsymbol{\lambda})^{-1} \circ \mathcal{A}(\boldsymbol{\lambda})^{\dagger} \circ \phi_{0}(x ; \boldsymbol{\lambda}+\boldsymbol{\delta}),
\end{aligned}
$$

with $\mathcal{E}_{n}(\boldsymbol{\lambda})=f_{n}(\boldsymbol{\lambda}) b_{n-1}(\boldsymbol{\lambda})$.

\section{A.1.1 Laguerre and Jacobi}

Operators $\mathcal{H}(\boldsymbol{\lambda}), \mathcal{A}(\boldsymbol{\lambda}), \mathcal{A}(\boldsymbol{\lambda})^{\dagger}, \mathcal{F}(\boldsymbol{\lambda})$ and $\mathcal{B}(\boldsymbol{\lambda})$ are

$$
\begin{aligned}
& \mathcal{H}(\boldsymbol{\lambda})=-\frac{d^{2}}{d x^{2}}+U(x ; \boldsymbol{\lambda}), \quad U(x ; \boldsymbol{\lambda})=\frac{\partial_{x}^{2} \phi_{0}(x ; \boldsymbol{\lambda})}{\phi_{0}(x ; \boldsymbol{\lambda})}, \\
& \mathcal{A}(\boldsymbol{\lambda}) \stackrel{\text { def }}{=} \frac{d}{d x}-\partial_{x} \log \left|\phi_{0}(x ; \boldsymbol{\lambda})\right|, \quad \mathcal{A}(\boldsymbol{\lambda})^{\dagger} \stackrel{\text { def }}{=}-\frac{d}{d x}-\partial_{x} \log \left|\phi_{0}(x ; \boldsymbol{\lambda})\right|,
\end{aligned}
$$




$$
\mathcal{F}(\boldsymbol{\lambda})=\left\{\begin{array}{ll}
2 \frac{d}{d \eta} & : \mathrm{L} \\
-4 \frac{d}{d \eta} & : \mathrm{J}
\end{array}, \quad \mathcal{B}(\boldsymbol{\lambda})=\left\{\begin{array}{ll}
-2 \eta \frac{d}{d \eta}-2\left(g+\frac{1}{2}-\eta\right) & : \mathrm{L} \\
\left(1-\eta^{2}\right) \frac{d}{d \eta}+h-g+(g+h+1) \eta & : \mathrm{J}
\end{array} .\right.\right.
$$

Various data of these systems are:

$$
\begin{array}{ll}
\mathrm{L}: \quad & 0<x<\infty, \quad \boldsymbol{\lambda}=g, \quad \boldsymbol{\delta}=1, \quad \kappa=1, \quad g>\frac{1}{2}, \\
& U(x ; \boldsymbol{\lambda})=x^{2}+\frac{g(g-1)}{x^{2}}-(1+2 g), \quad \eta(x)=x^{2}, \\
& \mathcal{E}_{n}(\boldsymbol{\lambda})=4 n, \quad f_{n}(\boldsymbol{\lambda})=-2, \quad b_{n-1}(\boldsymbol{\lambda})=-2 n, \\
& \phi_{0}(x ; \boldsymbol{\lambda})=e^{-\frac{1}{2} x^{2}} x^{g}, \quad P_{n}(\eta ; \boldsymbol{\lambda})=L_{n}^{\left(g-\frac{1}{2}\right)}(\eta), \\
& h_{n}(\boldsymbol{\lambda})=\frac{1}{2 n !} \Gamma\left(n+g+\frac{1}{2}\right), \\
\mathrm{J}: \quad 0<x<\frac{\pi}{2}, \quad \boldsymbol{\lambda}=(g, h), \quad \boldsymbol{\delta}=(1,1), \quad \kappa=1, \quad g, h>\frac{1}{2}, \\
& U(x ; \boldsymbol{\lambda})=\frac{g(g-1)}{\sin ^{2} x}+\frac{h(h-1)}{\cos ^{2} x}-(g+h)^{2}, \quad \eta(x)=\cos 2 x, \\
& \mathcal{E}_{n}(\boldsymbol{\lambda})=4 n(n+g+h), \quad f_{n}(\boldsymbol{\lambda})=-2(n+g+h), \quad b_{n-1}(\boldsymbol{\lambda})=-2 n, \\
& \phi_{0}(x ; \boldsymbol{\lambda})=(\sin x)^{g}(\cos x)^{h}, \quad P_{n}(\eta ; \boldsymbol{\lambda})=P_{n}^{\left(g-\frac{1}{2}, h-\frac{1}{2}\right)}(\eta), \\
& h_{n}(\boldsymbol{\lambda})=\frac{\Gamma\left(n+g+\frac{1}{2}\right) \Gamma\left(n+h+\frac{1}{2}\right)}{2 n !(2 n+g+h) \Gamma(n+g+h)},
\end{array}
$$

where $L_{n}^{(\alpha)}(\eta)$ and $P_{n}^{(\alpha, \beta)}(\eta)$ are the Laguerre and Jacobi polynomials [35] respectively.

\section{A.1.2 Wilson and Askey-Wilson}

Operators $\mathcal{H}(\boldsymbol{\lambda}), \mathcal{A}(\boldsymbol{\lambda}), \mathcal{A}(\boldsymbol{\lambda})^{\dagger}, \mathcal{F}(\boldsymbol{\lambda})$ and $\mathcal{B}(\boldsymbol{\lambda})$ are

$$
\begin{aligned}
& \mathcal{H}(\boldsymbol{\lambda})=\sqrt{V(x ; \boldsymbol{\lambda})} e^{\gamma p} \sqrt{V^{*}(x ; \boldsymbol{\lambda})}+\sqrt{V^{*}(x ; \boldsymbol{\lambda})} e^{-\gamma p} \sqrt{V(x ; \boldsymbol{\lambda})}-V(x ; \boldsymbol{\lambda})-V^{*}(x ; \boldsymbol{\lambda}), \\
& \mathcal{A}(\boldsymbol{\lambda}) \stackrel{\text { def }}{=} i\left(e^{\frac{\gamma}{2} p} \sqrt{V^{*}(x ; \boldsymbol{\lambda})}-e^{-\frac{\gamma}{2} p} \sqrt{V(x ; \boldsymbol{\lambda})}\right), \\
& \mathcal{A}(\boldsymbol{\lambda})^{\dagger} \stackrel{\text { def }}{=}-i\left(\sqrt{V(x ; \boldsymbol{\lambda})} e^{\frac{\gamma}{2} p}-\sqrt{V^{*}(x ; \boldsymbol{\lambda})} e^{-\frac{\gamma}{2} p}\right), \\
& \mathcal{F}(\boldsymbol{\lambda})=i \varphi(x)^{-1}\left(e^{\frac{\gamma}{2} p}-e^{-\frac{\gamma}{2} p}\right), \quad \mathcal{B}(\boldsymbol{\lambda})=-i\left(V(x ; \boldsymbol{\lambda}) e^{\frac{\gamma}{2} p}-V^{*}(x ; \boldsymbol{\lambda}) e^{-\frac{\gamma}{2} p}\right) \varphi(x) .
\end{aligned}
$$

Various data of these systems are:

$$
\begin{array}{ll}
\mathrm{W}: & 0<x<\infty, \quad \gamma=1, \quad \boldsymbol{\lambda}=\left(a_{1}, a_{2}, a_{3}, a_{4}\right), \quad \boldsymbol{\delta}=\left(\frac{1}{2}, \frac{1}{2}, \frac{1}{2}, \frac{1}{2}\right), \quad \kappa=1, \\
& V(x ; \boldsymbol{\lambda})=\frac{\prod_{j=1}^{4}\left(a_{j}+i x\right)}{2 i x(2 i x+1)}, \quad \eta(x)=x^{2}, \quad \varphi(x)=2 x, \\
& \mathcal{E}_{n}(\boldsymbol{\lambda})=n\left(n+b_{1}-1\right), \quad b_{1} \stackrel{\text { def }}{=} a_{1}+a_{2}+a_{3}+a_{4}, \\
& f_{n}(\boldsymbol{\lambda})=-n\left(n+b_{1}-1\right), \quad b_{n-1}(\boldsymbol{\lambda})=-1,
\end{array}
$$




$$
\begin{aligned}
& \phi_{0}(x ; \boldsymbol{\lambda})=\sqrt{\frac{\prod_{j=1}^{4} \Gamma\left(a_{j}+i x\right) \Gamma\left(a_{j}-i x\right)}{\Gamma(2 i x) \Gamma(-2 i x)}}, \\
& \check{P}_{n}(x ; \boldsymbol{\lambda})=P_{n}(\eta(x) ; \boldsymbol{\lambda})=W_{n}\left(\eta(x) ; a_{1}, a_{2}, a_{3}, a_{4}\right) \\
& \quad=\left(a_{1}+a_{2}, a_{1}+a_{3}, a_{1}+a_{4}\right)_{n}{ }_{4} F_{3}\left(\begin{array}{c}
-n, n+b_{1}-1, a_{1}+i x, a_{1}-i x \\
a_{1}+a_{2}, a_{1}+a_{3}, a_{1}+a_{4}
\end{array}\right. \\
& h_{n}(\boldsymbol{\lambda})=\frac{2 \pi n !\left(n+b_{1}-1\right)_{n} \prod_{1 \leq i<j \leq 4} \Gamma\left(n+a_{i}+a_{j}\right)}{\Gamma\left(2 n+b_{1}\right)},
\end{aligned}
$$

AW : $\quad 0<x<\pi, \quad \gamma=\log q, \quad q^{\boldsymbol{\lambda}}=\left(a_{1}, a_{2}, a_{3}, a_{4}\right), \quad \boldsymbol{\delta}=\left(\frac{1}{2}, \frac{1}{2}, \frac{1}{2}, \frac{1}{2}\right), \quad \kappa=q^{-1}$,

$$
\begin{aligned}
& V(x ; \boldsymbol{\lambda})=\frac{\prod_{j=1}^{4}\left(1-a_{j} e^{i x}\right)}{\left(1-e^{2 i x}\right)\left(1-q e^{2 i x}\right)}, \quad \eta(x)=\cos x, \quad \varphi(x)=2 \sin x, \\
& \mathcal{E}_{n}(\boldsymbol{\lambda})=\left(q^{-n}-1\right)\left(1-b_{4} q^{n-1}\right), \quad b_{4} \stackrel{\text { def }}{=} a_{1} a_{2} a_{3} a_{4}, \\
& f_{n}(\boldsymbol{\lambda})=q^{\frac{n}{2}}\left(q^{-n}-1\right)\left(1-b_{4} q^{n-1}\right), \quad b_{n-1}(\boldsymbol{\lambda})=q^{-\frac{n}{2}} \\
& \phi_{0}(x ; \boldsymbol{\lambda})=\sqrt{\frac{\left(e^{2 i x}, e^{-2 i x} ; q\right)_{\infty}}{\prod_{j=1}^{4}\left(a_{j} e^{i x}, a_{j} e^{-i x} ; q\right)_{\infty}}}, \\
& \check{P}_{n}(x ; \boldsymbol{\lambda})=P_{n}(\eta(x) ; \boldsymbol{\lambda})=p_{n}\left(\eta(x) ; a_{1}, a_{2}, a_{3}, a_{4} \mid q\right) \\
& =a_{1}^{-n}\left(a_{1} a_{2}, a_{1} a_{3}, a_{1} a_{4} ; q\right)_{n} \phi_{4}\left(q^{-n}, b_{4} q^{n-1}, a_{1} e^{i x}, a_{1} e^{-i x}\right. \\
& \left.a_{1} a_{2}, a_{1} a_{3}, a_{1} a_{4} \mid q\right), \\
& h_{n}(\boldsymbol{\lambda})=\frac{2 \pi\left(b_{4} q^{n-1} ; q\right)_{n}\left(b_{4} q^{2 n} ; q\right)_{\infty}}{\left(q^{n+1} ; q\right)_{\infty} \prod_{1 \leq i<j \leq 4}\left(a_{i} a_{j} q^{n} ; q\right)_{\infty}},
\end{aligned}
$$

where $W_{n}\left(\eta ; a_{1}, a_{2}, a_{3}, a_{4}\right)$ and $p_{n}\left(\eta ; a_{1}, a_{2}, a_{3}, a_{4} \mid q\right)$ are the Wilson and the Askey-Wilson polynomials [35] and $q^{\boldsymbol{\lambda}}$ stands for $q^{\left(\lambda_{1}, \lambda_{2}, \ldots\right)}=\left(q^{\lambda_{1}}, q^{\lambda_{2}}, \ldots\right)$ and $0<q<1$. The parameters are restricted by

$$
\left\{a_{1}^{*}, a_{2}^{*}, a_{3}^{*}, a_{4}^{*}\right\}=\left\{a_{1}, a_{2}, a_{3}, a_{4}\right\} \quad \text { (as a set); W: Re } a_{j}>0, \quad \text { AW }:\left|a_{j}\right|<1 .
$$

\section{A.2 Virtual state wavefunctions}

We have two types of virtual states, type I $\tilde{\phi}_{\mathrm{v}}^{\mathrm{I}}(x ; \boldsymbol{\lambda})$ and type II $\tilde{\phi}_{\mathrm{v}}^{\mathrm{II}}(x ; \boldsymbol{\lambda})\left(\mathrm{v} \in \mathbb{Z}_{\geq 0}\right)$.

\section{A.2.1 Laguerre and Jacobi}

$\mathrm{L} 1: \quad \tilde{\phi}_{\mathrm{v}}^{\mathrm{I}}(x ; \boldsymbol{\lambda}) \stackrel{\text { def }}{=} i^{-g} \phi_{\mathrm{v}}\left(i x ; \mathfrak{t}^{\mathrm{I}}(\boldsymbol{\lambda})\right), \quad \xi_{\mathrm{v}}^{\mathrm{I}}(\eta ; \boldsymbol{\lambda}) \stackrel{\text { def }}{=} P_{\mathrm{v}}\left(-\eta ; \mathfrak{t}^{\mathrm{I}}(\boldsymbol{\lambda})\right)$,

$\mathfrak{t}^{\mathrm{I}} \stackrel{\text { def }}{=} \mathrm{id}, \quad \tilde{\boldsymbol{\delta}}^{\mathrm{I}} \stackrel{\text { def }}{=} 1, \quad \tilde{\mathcal{E}}_{\mathrm{v}}^{\mathrm{I}}(\boldsymbol{\lambda})=-4\left(g+\mathrm{v}+\frac{1}{2}\right)$,

L2 : $\quad \tilde{\phi}_{\mathrm{v}}^{\mathrm{II}}(x ; \boldsymbol{\lambda}) \stackrel{\text { def }}{=} \phi_{\mathrm{v}}\left(x ; \mathfrak{t}^{\mathrm{II}}(\boldsymbol{\lambda})\right), \quad \xi_{\mathrm{v}}^{\mathrm{II}}(\eta ; \boldsymbol{\lambda}) \stackrel{\text { def }}{=} P_{\mathrm{v}}\left(\eta ; \mathfrak{t}^{\mathrm{II}}(\boldsymbol{\lambda})\right)$,

$\mathfrak{t}^{\mathrm{II}}(\boldsymbol{\lambda}) \stackrel{\text { def }}{=} 1-g, \quad \tilde{\boldsymbol{\delta}}^{\mathrm{II}} \stackrel{\text { def }}{=}-1, \quad \tilde{\mathcal{E}}_{\mathrm{v}}^{\mathrm{II}}(\boldsymbol{\lambda})=-4\left(g-\mathrm{v}-\frac{1}{2}\right)$, 
$\mathrm{J} 1: \quad \tilde{\phi}_{\mathrm{v}}^{\mathrm{I}}(x ; \boldsymbol{\lambda}) \stackrel{\text { def }}{=} \phi_{\mathrm{v}}\left(x ; \mathfrak{t}^{\mathrm{I}}(\boldsymbol{\lambda})\right), \quad \xi_{\mathrm{v}}^{\mathrm{I}}(\eta ; \boldsymbol{\lambda}) \stackrel{\text { def }}{=} P_{\mathrm{v}}\left(\eta ; \mathfrak{t}^{\mathrm{I}}(\boldsymbol{\lambda})\right)$,

$$
\mathfrak{t}^{\mathrm{I}}(\boldsymbol{\lambda}) \stackrel{\text { def }}{=}(g, 1-h), \quad \tilde{\boldsymbol{\delta}}^{\mathrm{I}} \stackrel{\text { def }}{=}(1,-1), \quad \tilde{\mathcal{E}}_{\mathrm{v}}^{\mathrm{I}}(\boldsymbol{\lambda})=-4\left(g+\mathrm{v}+\frac{1}{2}\right)\left(h-\mathrm{v}-\frac{1}{2}\right),
$$

$\mathrm{J} 2: \quad \tilde{\phi}_{\mathrm{v}}^{\mathrm{II}}(x ; \boldsymbol{\lambda}) \stackrel{\text { def }}{=} \phi_{\mathrm{v}}\left(x ; \mathfrak{t}^{\mathrm{II}}(\boldsymbol{\lambda})\right), \quad \xi_{\mathrm{v}}^{\mathrm{II}}(\eta ; \boldsymbol{\lambda}) \stackrel{\text { def }}{=} P_{\mathrm{v}}\left(\eta ; \mathfrak{t}^{\mathrm{II}}(\boldsymbol{\lambda})\right)$,

$$
\mathfrak{t}^{\mathrm{II}}(\boldsymbol{\lambda}) \stackrel{\text { def }}{=}(1-g, h), \quad \tilde{\boldsymbol{\delta}}^{\mathrm{II}} \stackrel{\text { def }}{=}(-1,1), \quad \tilde{\mathcal{E}}_{\mathrm{v}}^{\mathrm{II}}(\boldsymbol{\lambda})=-4\left(g-\mathrm{v}-\frac{1}{2}\right)\left(h+\mathrm{v}+\frac{1}{2}\right) .
$$

We have changed the signs of $\tilde{\boldsymbol{\delta}}^{\mathrm{I}}$ and $\tilde{\boldsymbol{\delta}}^{\mathrm{II}}$ from those in [5].

\section{A.2.2 Wilson and Askey-Wilson}

type I : $\quad \tilde{\phi}_{\mathrm{v}}^{\mathrm{I}}(x ; \boldsymbol{\lambda}) \stackrel{\text { def }}{=} \phi_{\mathrm{v}}\left(x ; \mathfrak{t}^{\mathrm{I}}(\boldsymbol{\lambda})\right), \quad \xi_{\mathrm{v}}^{\mathrm{I}}(\eta ; \boldsymbol{\lambda}) \stackrel{\text { def }}{=} P_{\mathrm{v}}\left(\eta ; \mathfrak{t}^{\mathrm{I}}(\boldsymbol{\lambda})\right), \quad \dot{\xi}_{\mathrm{v}}^{\mathrm{I}}(x ; \boldsymbol{\lambda}) \stackrel{\text { def }}{=} \xi_{\mathrm{v}}^{\mathrm{I}}(\eta(x) ; \boldsymbol{\lambda})$,

$$
\begin{aligned}
& \mathfrak{t}^{\mathrm{I}}(\boldsymbol{\lambda}) \stackrel{\text { def }}{=}\left(1-\lambda_{1}, 1-\lambda_{2}, \lambda_{3}, \lambda_{4}\right), \quad \tilde{\boldsymbol{\delta}}^{\mathrm{I}} \stackrel{\text { def }}{=}\left(-\frac{1}{2},-\frac{1}{2}, \frac{1}{2}, \frac{1}{2}\right), \\
& \tilde{\mathcal{E}}_{\mathrm{v}}^{\mathrm{I}}(\boldsymbol{\lambda})=\left\{\begin{array}{ll}
-\left(a_{1}+a_{2}-\mathrm{v}-1\right)\left(a_{3}+a_{4}+\mathrm{v}\right) & : \mathrm{W} \\
-\left(1-a_{1} a_{2} q^{-\mathrm{v}-1}\right)\left(1-a_{3} a_{4} q^{\mathrm{v}}\right) & : \mathrm{AW}
\end{array}, \quad \alpha^{\mathrm{I}}(\boldsymbol{\lambda})=\left\{\begin{array}{ll}
1 & : \mathrm{W} \\
a_{1} a_{2} q^{-1} & : \mathrm{AW}
\end{array},\right.\right.
\end{aligned}
$$

type II : $\quad \tilde{\phi}_{\mathrm{v}}^{\mathrm{II}}(x ; \boldsymbol{\lambda}) \stackrel{\text { def }}{=} \phi_{\mathrm{v}}\left(x ; \mathfrak{t}^{\mathrm{II}}(\boldsymbol{\lambda})\right), \quad \xi_{\mathrm{v}}^{\mathrm{II}}(\eta ; \boldsymbol{\lambda}) \stackrel{\text { def }}{=} P_{\mathrm{v}}\left(\eta ; \mathfrak{t}^{\mathrm{II}}(\boldsymbol{\lambda})\right), \quad \dot{\xi}_{\mathrm{v}}^{\mathrm{II}}(x ; \boldsymbol{\lambda}) \stackrel{\text { def }}{=} \xi_{\mathrm{v}}^{\mathrm{II}}(\eta(x) ; \boldsymbol{\lambda})$,

$$
\begin{aligned}
& \mathfrak{t}^{\mathrm{II}}(\boldsymbol{\lambda}) \stackrel{\text { def }}{=}\left(\lambda_{1}, \lambda_{2}, 1-\lambda_{3}, 1-\lambda_{4}\right), \quad \tilde{\boldsymbol{\delta}}^{\mathrm{II}} \stackrel{\text { def }}{=}\left(\frac{1}{2}, \frac{1}{2},-\frac{1}{2},-\frac{1}{2}\right), \\
& \tilde{\mathcal{E}}_{\mathrm{v}}^{\mathrm{II}}(\boldsymbol{\lambda})=\left\{\begin{array}{ll}
-\left(a_{3}+a_{4}-\mathrm{v}-1\right)\left(a_{1}+a_{2}+\mathrm{v}\right) & : \mathrm{W} \\
-\left(1-a_{3} a_{4} q^{-\mathrm{v}-1}\right)\left(1-a_{1} a_{2} q^{\mathrm{v}}\right) & : \mathrm{AW}
\end{array}, \quad \alpha^{\mathrm{II}}(\boldsymbol{\lambda})=\left\{\begin{array}{ll}
1 & : \mathrm{W} \\
a_{3} a_{4} q^{-1} & : \mathrm{AW}
\end{array} .\right.\right.
\end{aligned}
$$

\section{A.3 Pseudo virtual state wavefunctions}

The twist operation $\mathfrak{t}$ is defined by

$$
\mathfrak{t} \stackrel{\text { def }}{=} \mathfrak{t}^{\mathrm{II}} \circ \mathfrak{t}^{\mathrm{I}}\left(\Rightarrow \mathfrak{t}=\mathfrak{t}^{\mathrm{I}} \circ \mathfrak{t}^{\mathrm{II}}\right),
$$

and the energy of pseudo virtual state $\tilde{\phi}_{\mathrm{v}}^{\text {pvs }}(x ; \boldsymbol{\lambda})\left(\mathrm{v} \in \mathbb{Z}_{\geq 0}\right)$ is

$$
\tilde{\mathcal{E}}_{\mathrm{v}}^{\mathrm{pvs}}(\boldsymbol{\lambda})=\mathcal{E}_{-\mathrm{v}-1}(\boldsymbol{\lambda}) .
$$

\section{A.3.1 Laguerre and Jacobi}

$$
\begin{array}{ll}
\mathrm{L}: & \tilde{\phi}_{\mathrm{v}}^{\text {pvs }}(x ; \boldsymbol{\lambda}) \stackrel{\text { def }}{=} i^{g-1} \phi_{\mathrm{v}}(i x ; \mathfrak{t}(\boldsymbol{\lambda})), \quad \xi_{\mathrm{v}}^{\text {pvs }}(\eta ; \boldsymbol{\lambda}) \stackrel{\text { def }}{=} P_{\mathrm{v}}(-\eta ; \mathfrak{t}(\boldsymbol{\lambda})), \quad \mathfrak{t}(\boldsymbol{\lambda})=1-g, \\
\mathrm{~J}: & \tilde{\phi}_{\mathrm{v}}^{\text {pvs }}(x ; \boldsymbol{\lambda}) \stackrel{\text { def }}{=} \phi_{\mathrm{v}}(x ; \mathfrak{t}(\boldsymbol{\lambda})), \quad \xi_{\mathrm{v}}^{\text {pvs }}(\eta ; \boldsymbol{\lambda}) \stackrel{\text { def }}{=} P_{\mathrm{v}}(\eta ; \mathfrak{t}(\boldsymbol{\lambda})), \quad \mathfrak{t}(\boldsymbol{\lambda})=(1-g, 1-h) .
\end{array}
$$

\section{A.3.2 Wilson and Askey-Wilson}

$$
\begin{aligned}
& \tilde{\phi}_{\mathrm{v}}^{\text {pvs }}(x ; \boldsymbol{\lambda}) \stackrel{\text { def }}{=} \phi_{\mathrm{v}}(x ; \mathfrak{t}(\boldsymbol{\lambda})), \quad \xi_{\mathrm{v}}^{\text {pvs }}(\eta ; \boldsymbol{\lambda}) \stackrel{\text { def }}{=} P_{\mathrm{v}}(\eta ; \mathfrak{t}(\boldsymbol{\lambda})), \\
& \mathfrak{t}(\boldsymbol{\lambda})=\left(1-\lambda_{1}, 1-\lambda_{2}, 1-\lambda_{3}, 1-\lambda_{4}\right) .
\end{aligned}
$$




\section{A.4 Multi-indexed orthogonal polynomials}

The set $\mathcal{D}$ is given in (2.11). The eigenfunctions $\phi_{\mathcal{D} n}(x ; \boldsymbol{\lambda})$ of the deformed system $\mathcal{H}_{\mathcal{D}}(\boldsymbol{\lambda})$ have the form (2.16)-(2.19) . The denominator polynomial $\Xi_{\mathcal{D}}(\eta ; \boldsymbol{\lambda})$ and the multi-indexed orthogonal polynomial $P_{\mathcal{D}, n}(\eta ; \boldsymbol{\lambda})$ are polynomials in $\eta$ and their degree are generically $\ell_{\mathcal{D}}$ and $\ell_{\mathcal{D}}+n$, respectively $\left(\ell_{\mathcal{D}}\right.$ is given by $\left.(2.20)\right)$.

\section{A.4.1 Laguerre and Jacobi}

$$
\begin{aligned}
& \Xi_{\mathcal{D}}(\eta ; \boldsymbol{\lambda}) \stackrel{\text { def }}{=} \mathrm{W}\left[\mu_{1}, \ldots, \mu_{M_{\mathrm{I}}}, \nu_{1}, \ldots, \nu_{M_{\mathrm{II}}}\right](\eta) \\
& \times\left\{\begin{array}{ll}
e^{-M_{\mathrm{I}} \eta} \eta^{\left(M_{\mathrm{I}}+g-\frac{1}{2}\right) M_{\mathrm{II}}} & : \mathrm{L} \\
\left(\frac{1-\eta}{2}\right)^{\left(M_{\mathrm{I}}+g-\frac{1}{2}\right) M_{\mathrm{II}}}\left(\frac{1+\eta}{2}\right)^{\left(M_{\mathrm{II}}+h-\frac{1}{2}\right) M_{\mathrm{I}}} & : \mathrm{J}
\end{array},\right. \\
& P_{\mathcal{D}, n}(\eta ; \boldsymbol{\lambda}) \stackrel{\text { def }}{=} \mathrm{W}\left[\mu_{1}, \ldots, \mu_{M_{\mathrm{I}}}, \nu_{1}, \ldots, \nu_{M_{\mathrm{II}}}, P_{n}\right](\eta) \\
& \times\left\{\begin{array}{ll}
e^{-M_{\mathrm{I}} \eta} \eta^{\left(M_{\mathrm{I}}+g+\frac{1}{2}\right) M_{\mathrm{II}}} & : \mathrm{L} \\
\left(\frac{1-\eta}{2}\right)^{\left(M_{\mathrm{I}}+g+\frac{1}{2}\right) M_{\mathrm{II}}}\left(\frac{1+\eta}{2}\right)^{\left(M_{\mathrm{II}}+h+\frac{1}{2}\right) M_{\mathrm{I}}} & : \mathrm{J}
\end{array},\right. \\
& \mu_{j}=\xi_{d_{j}^{\mathrm{I}}}^{\mathrm{I}}(\eta ; \boldsymbol{\lambda}) \times\left\{\begin{array}{ll}
e^{\eta} & : \mathrm{L} \\
\left(\frac{1+\eta}{2}\right)^{\frac{1}{2}-h} & : \mathrm{J}
\end{array}, \quad \nu_{j}=\xi_{d_{j}^{\mathrm{II}}}^{\mathrm{II}}(\eta ; \boldsymbol{\lambda}) \times\left\{\begin{array}{ll}
\eta^{\frac{1}{2}-g} & : \mathrm{L} \\
\left(\frac{1-\eta}{2}\right)^{\frac{1}{2}-g} & : \mathrm{J}
\end{array} .\right.\right.
\end{aligned}
$$

\section{A.4.2 Wilson and Askey-Wilson}

$$
\begin{aligned}
& \check{\Xi}_{\mathcal{D}}(x ; \boldsymbol{\lambda}) \stackrel{\text { def }}{=} \Xi_{\mathcal{D}}(\eta(x) ; \boldsymbol{\lambda}), \quad \check{P}_{\mathcal{D}, n}(x ; \boldsymbol{\lambda}) \stackrel{\text { def }}{=} P_{\mathcal{D}, n}(\eta(x) ; \boldsymbol{\lambda}), \\
& \check{\Xi}_{\mathcal{D}}(x ; \boldsymbol{\lambda}) \stackrel{\text { def }}{=} A^{-1} \varphi_{M}(x)^{-1} i^{\frac{1}{2} M(M-1)}\left|\begin{array}{llllll}
\vec{X}_{d_{1}^{\perp}}^{(M)} & \cdots & \vec{X}_{d_{M_{\mathrm{I}}}^{\mathrm{N}}}^{(M)} & \vec{Y}_{d_{1}^{\mathrm{II}}}^{(M)} & \ldots & \vec{Y}_{d_{M_{\mathrm{II}}}^{\mathrm{II}}}^{(M)}
\end{array}\right|, \\
& A=\left\{\begin{array}{l}
\prod_{k=3,4} \prod_{j=1}^{M_{\mathrm{I}}-1}\left(a_{k}-\frac{M-1}{2}+i x, a_{k}-\frac{M-1}{2}-i x\right)_{j} \\
\quad \times \prod_{k=1,2} \prod_{j=1}^{M_{\mathrm{II}}-1}\left(a_{k}-\frac{M-1}{2}+i x, a_{k}-\frac{M-1}{2}-i x\right)_{j} \\
\prod_{k=3,4} \prod_{j=1}^{M_{\mathrm{I}}-1} a_{k}^{-j} q^{\frac{1}{4} j(j+1)}\left(a_{k} q^{-\frac{M-1}{2}} e^{i x}, a_{k} q^{-\frac{M-1}{2}} e^{-i x} ; q\right)_{j} \\
\quad \times \prod_{k=1,2} \prod_{j=1}^{M_{\mathrm{II}}-1} a_{k}^{-j} q^{\frac{1}{4} j(j+1)}\left(a_{k} q^{-\frac{M-1}{2}} e^{i x}, a_{k} q^{-\frac{M-1}{2}} e^{-i x} ; q\right)_{j} \quad: \mathrm{AW}
\end{array}\right. \\
& \check{P}_{\mathcal{D}, n}(x ; \boldsymbol{\lambda}) \stackrel{\text { def }}{=} B^{-1} \varphi_{M+1}(x)^{-1} \\
& \times i^{\frac{1}{2} M(M+1)}\left|\begin{array}{lllllll}
\vec{X}_{d_{1}^{\mathrm{I}}}^{(M+1)} & \cdots & \vec{X}_{d_{M_{\mathrm{I}}}^{\mathrm{M}}}^{(M+1)} & \vec{Y}_{d_{1}^{\mathrm{II}}}^{(M+1)} & \cdots & \vec{Y}_{d_{M_{I I}}^{\mathrm{II}}}^{(M+1)} & \vec{Z}_{n}^{(M+1)}
\end{array}\right|, \\
& B=\left\{\begin{array}{ll}
\prod_{k=3,4} \prod_{j=1}^{M_{\mathrm{I}}}\left(a_{k}-\frac{M}{2}+i x, a_{k}-\frac{M}{2}-i x\right)_{j} & \\
\quad \times \prod_{k=1,2} \prod_{j=1}^{M_{\mathrm{II}}}\left(a_{k}-\frac{M}{2}+i x, a_{k}-\frac{M}{2}-i x\right)_{j} & : \mathrm{W} \\
\prod_{k=3,4} \prod_{j=1}^{M_{\mathrm{I}}} a_{k}^{-j} q^{\frac{1}{4} j(j+1)}\left(a_{k} q^{-\frac{M}{2}} e^{i x}, a_{k} q^{-\frac{M}{2}} e^{-i x} ; q\right)_{j} & \\
\quad & \times \prod_{k=1,2} \prod_{j=1}^{M_{\mathrm{II}}} a_{k}^{-j} q^{\frac{1}{4} j(j+1)}\left(a_{k} q^{-\frac{M}{2}} e^{i x}, a_{k} q^{-\frac{M}{2}} e^{-i x} ; q\right)_{j}: \mathrm{AW}
\end{array},\right.
\end{aligned}
$$


where

$$
\begin{aligned}
& \left(\vec{X}_{\mathrm{v}}^{(M)}\right)_{j}=r_{j}^{\mathrm{II}}\left(x_{j}^{(M)} ; \boldsymbol{\lambda}, M\right) \check{\xi}_{\mathrm{v}}^{\mathrm{I}}\left(x_{j}^{(M)} ; \boldsymbol{\lambda}\right), \quad(1 \leq j \leq M), \\
& \left(\vec{Y}_{\mathrm{v}}^{(M)}\right)_{j}=r_{j}^{\mathrm{I}}\left(x_{j}^{(M)} ; \boldsymbol{\lambda}, M\right) \check{\xi}_{\mathrm{v}}^{\mathrm{II}}\left(x_{j}^{(M)} ; \boldsymbol{\lambda}\right), \\
& \left(\vec{Z}_{n}^{(M)}\right)_{j}=r_{j}^{\mathrm{II}}\left(x_{j}^{(M)} ; \boldsymbol{\lambda}, M\right) r_{j}^{\mathrm{I}}\left(x_{j}^{(M)} ; \boldsymbol{\lambda}, M\right) \check{P}_{n}\left(x_{j}^{(M)} ; \boldsymbol{\lambda}\right),
\end{aligned}
$$

and $x_{j}^{(n)}=x+i\left(\frac{n+1}{2}-j\right) \gamma$ and

$$
\begin{aligned}
r_{j}^{\mathrm{I}}\left(x_{j}^{(M)} ; \boldsymbol{\lambda}, M\right)= & \alpha^{\mathrm{I}}\left(\boldsymbol{\lambda}+(M-1) \tilde{\boldsymbol{\delta}}^{\mathrm{I}}\right)^{-\frac{1}{2}(M-1)} \kappa^{\frac{1}{2}(M-1)^{2}-(j-1)(M-j)} \\
& \times\left\{\begin{array}{l}
\prod_{k=1,2}\left(a_{k}-\frac{M-1}{2}+i x\right)_{j-1}\left(a_{k}-\frac{M-1}{2}-i x\right)_{M-j} \\
e^{i x(M+1-2 j)} \prod_{k=1,2}\left(a_{k} q^{-\frac{M-1}{2}} e^{i x} ; q\right)_{j-1}\left(a_{k} q^{-\frac{M-1}{2}} e^{-i x} ; q\right)_{M-j}
\end{array}\right. \\
r_{j}^{\mathrm{II}}\left(x_{j}^{(M)} ; \boldsymbol{\lambda}, M\right)= & \alpha^{\mathrm{II}}\left(\boldsymbol{\lambda}+(M-1) \tilde{\boldsymbol{\delta}}^{\mathrm{II}}\right)^{-\frac{1}{2}(M-1)} \kappa^{\frac{1}{2}(M-1)^{2}-(j-1)(M-j)} \\
& \times\left\{\begin{array}{l}
\prod_{k=3,4}\left(a_{k}-\frac{M-1}{2}+i x\right)_{j-1}\left(a_{k}-\frac{M-1}{2}-i x\right)_{M-j} \\
e^{i x(M+1-2 j)} \prod_{k=3,4}\left(a_{k} q^{-\frac{M-1}{2}} e^{i x} ; q\right)_{j-1}\left(a_{k} q^{-\frac{M-1}{2}} e^{-i x} ; q\right)_{M-j}
\end{array}\right.
\end{aligned}
$$

The auxiliary function $\varphi_{M}(x)$ is defined by

$$
\varphi_{M}(x) \stackrel{\text { def }}{=} \varphi(x)^{\left[\frac{M}{2}\right]} \prod_{k=1}^{M-2}\left(\varphi\left(x-i \frac{k}{2} \gamma\right) \varphi\left(x+i \frac{k}{2} \gamma\right)\right)^{\left[\frac{M-k}{2}\right]},
$$

and $\varphi_{0}(x)=\varphi_{1}(x)=1$, see [21]. Here $[x]$ denotes the greatest integer not exceeding $x$.

\section{A.4.3 coefficients of the highest degree terms}

The coefficients of the highest degree term of the polynomials $\Xi_{\mathcal{D}}$ and $P_{\mathcal{D}, n}$ are

$$
\begin{aligned}
& \Xi_{\mathcal{D}}(\eta ; \boldsymbol{\lambda})=c_{\mathcal{D}}^{\Xi}(\boldsymbol{\lambda}) \eta^{\ell_{\mathcal{D}}}+(\text { lower order terms }), \\
& P_{\mathcal{D}}(\eta ; \boldsymbol{\lambda})=c_{\mathcal{D}, n}^{P}(\boldsymbol{\lambda}) \eta^{\ell_{\mathcal{D}}+n}+(\text { lower order terms), } \\
& c_{\mathcal{D}}^{\Xi}(\boldsymbol{\lambda})=\prod_{j=1}^{M_{\mathrm{I}}} c_{d_{j}^{\mathrm{I}}}^{\mathrm{I}}(\boldsymbol{\lambda}) \cdot \prod_{j=1}^{M_{\mathrm{II}}} c_{d_{j}^{\mathrm{II}}}^{\mathrm{II}}(\boldsymbol{\lambda}) \\
& \times \begin{cases}\prod_{1 \leq j<k \leq M_{\mathrm{I}}}\left(d_{k}^{\mathrm{I}}-d_{j}^{\mathrm{I}}\right) \cdot \prod_{1 \leq j<k \leq M_{\mathrm{II}}}\left(d_{k}^{\mathrm{II}}-d_{j}^{\mathrm{II}}\right) & : \mathrm{L}, \mathrm{J}, \mathrm{W} \\
\prod_{1 \leq j<k \leq M_{\mathrm{I}}} \frac{1}{2} q^{\frac{1}{2}\left(d_{j}^{\mathrm{I}}-d_{k}^{\mathrm{I}}\right)}\left(1-q^{d_{k}^{\mathrm{I}}-d_{j}^{\mathrm{I}}}\right) \cdot \prod_{1 \leq j<k \leq M_{\mathrm{II}}} \frac{1}{2} q^{\frac{1}{2}\left(d_{j}^{\mathrm{II}}-d_{k}^{\mathrm{II}}\right)}\left(1-q^{\left.d_{k}^{\mathrm{II}}-d_{j}^{\mathrm{II}}\right)}\right. & : \mathrm{AW}\end{cases}
\end{aligned}
$$




$$
\begin{aligned}
& \times \begin{cases}(-1)^{M_{\mathrm{I}} M_{\mathrm{II}}} & : \mathrm{L} \\
\prod_{j=1}^{M_{\mathrm{I}}} \prod_{k=1}^{M_{\mathrm{II}}} \frac{1}{4}\left(g-h+d_{j}^{\mathrm{I}}-d_{k}^{\mathrm{II}}\right) & : \mathrm{J} \\
\prod_{j=1}^{M_{\mathrm{I}}} \prod_{k=1}^{M_{\mathrm{II}}}\left(-a_{3}-a_{4}-d_{j}^{\mathrm{I}}+a_{1}+a_{2}+d_{k}^{\mathrm{II}}\right) & : \mathrm{W} \\
\prod_{j=1}^{M_{\mathrm{I}}} \prod_{k=1}^{M_{\mathrm{II}}} \frac{2}{\sqrt{a_{1} a_{2} a_{3} a_{4}}} q^{j+k-2-\frac{1}{2}\left(d_{j}^{\mathrm{I}}+d_{k}^{\mathrm{II}}\right)}\left(a_{3} a_{4} q^{d_{j}^{\mathrm{I}}}-a_{1} a_{2} q^{d_{k}^{\mathrm{II}}}\right) & : \mathrm{AW}\end{cases} \\
& c_{\mathcal{D}, n}^{P}(\boldsymbol{\lambda})=c_{\mathcal{D}}^{\Xi}(\boldsymbol{\lambda}) c_{n}(\boldsymbol{\lambda}) \\
& \times \begin{cases}(-1)^{M_{\mathrm{I}}} \prod_{j=1}^{M_{\mathrm{II}}}\left(g+n-d_{j}^{\mathrm{II}}-\frac{1}{2}\right) & : \mathrm{L} \\
\prod_{j=1}^{M_{\mathrm{I}}} \frac{1}{2}\left(h+n-d_{j}^{\mathrm{I}}-\frac{1}{2}\right) \cdot \prod_{j=1}^{M_{\mathrm{II}}} \frac{-1}{2}\left(g+n-d_{j}^{\mathrm{II}}-\frac{1}{2}\right) & : \mathrm{J} \\
\prod_{j=1}^{M_{\mathrm{I}}}\left(-a_{1}-a_{2}-n+d_{j}^{\mathrm{I}}+1\right) \cdot \prod_{j=1}^{M_{\mathrm{II}}}\left(-a_{3}-a_{4}-n+d_{j}^{\mathrm{II}}+1\right) & : \mathrm{W} \\
q^{2 M_{\mathrm{I}} M_{\mathrm{II}}} \prod_{j=1}^{M_{\mathrm{I}}}\left(a_{1} a_{2}\right)^{-\frac{1}{2}} q^{\frac{1}{2}\left(d_{j}^{\mathrm{I}}+1-n\right)}\left(1-a_{1} a_{2} q^{n-d_{j}^{\mathrm{I}}-1}\right) & \\
\quad \times \prod_{j=1}^{M_{\mathrm{II}}}\left(a_{3} a_{4}\right)^{-\frac{1}{2}} q^{\frac{1}{2}\left(d_{j}^{\mathrm{II}}+1-n\right)}\left(1-a_{3} a_{4} q^{n-d_{j}^{\mathrm{II}}-1}\right) & : \mathrm{AW}\end{cases}
\end{aligned}
$$

where $c_{n}, c_{n}^{\mathrm{I}}$ and $c_{n}^{\mathrm{II}}$ are

$$
\begin{aligned}
& P_{n}(\eta ; \boldsymbol{\lambda})=c_{n}(\boldsymbol{\lambda}) \eta^{n}+(\text { lower order terms }), \\
& c_{n}(\boldsymbol{\lambda})=\left\{\begin{array}{ll}
\frac{(-1)^{n}}{n !} & : \mathrm{L} \\
\frac{(n+g+h)_{n}}{2^{n} n !} & : \mathrm{J}
\end{array}, \quad c_{n}(\boldsymbol{\lambda})=\left\{\begin{array}{ll}
(-1)^{n}\left(n+b_{1}-1\right)_{n} & : \mathrm{W} \\
2^{n}\left(b_{4} q^{n-1} ; q\right)_{n} & : \mathrm{AW}
\end{array},\right.\right. \\
& c_{\mathrm{v}}^{\mathrm{I}(\boldsymbol{\lambda}) \stackrel{\text { def }}{=}\left\{\begin{array}{ll}
(-1)^{\mathrm{v}} c_{\mathrm{v}}(\boldsymbol{\lambda}) & : \mathrm{L} \\
c_{\mathrm{v}}\left(\mathfrak{t}^{\mathrm{I}}(\boldsymbol{\lambda})\right) & : \mathrm{J}, \mathrm{W}, \mathrm{AW}
\end{array}, \quad c_{\mathrm{v}}^{\mathrm{II}}(\boldsymbol{\lambda}) \stackrel{\text { def }}{=} c_{\mathrm{v}}\left(\mathfrak{t}^{\mathrm{II}}(\boldsymbol{\lambda})\right): \mathrm{L}, \mathrm{J}, \mathrm{W}, \mathrm{AW} .\right.}
\end{aligned}
$$

\section{References}

[1] D. Gómez-Ullate, N. Kamran and R. Milson, "An extension of Bochner's problem: exceptional invariant subspaces," J. Approx Theory 162 (2010) 987-1006, arXiv:0805. 3376 [math-ph]; "An extended class of orthogonal polynomials defined by a SturmLiouville problem," J. Math. Anal. Appl. 359 (2009) 352-367, arXiv:0807.3939][math$\mathrm{ph}$.

[2] C. Quesne, "Exceptional orthogonal polynomials, exactly solvable potentials and supersymmetry," J. Phys. A41 (2008) 392001 (6pp), arXiv:0807.4087[quant-ph].

[3] S. Odake and R. Sasaki, "Infinitely many shape invariant potentials and new orthogonal polynomials," Phys. Lett. B679 (2009) 414-417, arXiv:0906.0142[math-ph]; "Another set of infinitely many exceptional $\left(X_{\ell}\right)$ Laguerre polynomials," Phys. Lett. B684 (2010) 173-176, arXiv:0911.3442[math-ph] . 
[4] D. Gómez-Ullate, N. Kamran and R. Milson, "Two-step Darboux transformations and exceptional Laguerre polynomials," J. Math. Anal. Appl. 387 (2012) 410-418, arXiv: 1103.5724 [math-ph].

[5] S. Odake and R.Sasaki, "Exactly solvable quantum mechanics and infinite families of multi-indexed orthogonal polynomials," Phys. Lett. B702 (2011) 164-170, arXiv: 1105.0508 [math-ph].

[6] S. Odake and R. Sasaki, "Infinitely many shape invariant potentials and cubic identities of the Laguerre and Jacobi polynomials," J. Math. Phys. 51 (2010) 053513 (9pp), arXiv:0911.1585[math-ph].

[7] C.-L.Ho, S. Odake and R. Sasaki, "Properties of the exceptional $\left(X_{\ell}\right)$ Laguerre and Jacobi polynomials," SIGMA 7 (2011) 107 (24pp), arXiv:0912.5447[math-ph].

[8] D. Gómez-Ullate, N. Kamran and R. Milson, "Exceptional orthogonal polynomials and the Darboux transformation," J. Phys. A43 (2010) 434016, arXiv:1002.2666[math$\mathrm{ph}$.

[9] R. Sasaki, S. Tsujimoto and A.Zhedanov, "Exceptional Laguerre and Jacobi polynomials and the corresponding potentials through Darboux-Crum transformations," J. Phys. A43 (2010) 315204, arXiv:1004.4711[math-ph].

[10] D. Gómez-Ullate, N. Kamran and R. Milson, "On orthogonal polynomials spanning a non-standard flag," Contemp. Math. 563 (2011) 51-72, arXiv:1101.5584[math-ph].

[11] D. Gómez-Ullate, N. Kamran and R. Milson, "A conjecture on exceptional orthogonal polynomials," Found. Comput. Math. 13 (2013) 615-666, arXiv:1203.6857[math-ph] .

[12] R. Sasaki and K. Takemura, "Global solutions of certain second order differential equations with a high degree of apparent singularity," SIGMA 8 (2012) 085 (18pp), arXiv: $1207.5302[$ math. CA] .

[13] C.-L. Ho, R. Sasaki and K. Takemura, "Confluence of apparent singularities in multiindexed orthogonal polynomials: the Jacobi case," J. Phys. A46 (2013) 115205 (21pp), arXiv:1210.0207[math.CA] . 
[14] C. Quesne, "Solvable rational potentials and exceptional orthogonal polynomials in supersymmetric quantum mechanics," SIGMA 5 (2009) 084 (24pp), arXiv:0906.2331 [math-ph].

[15] S. Odake and R. Sasaki, "A new family of shape invariantly deformed Darboux-PöschlTeller potentials with continuous $\ell$," J. Phys. A44 (2011) 195203 (14pp), arXiv:1007. $3800[$ math-ph].

[16] Y. Grandati, "Solvable rational extensions of the isotonic oscillator," Ann. Phys. 326 (2011) 2074-2090, arXiv:1101.0055[math-ph]; "Multistep DBT and regular rational extensions of the isotonic oscillator," Ann. Phys. 327 (2012) 2411-2431, arXiv:1108. 4503 [math-ph].

[17] C-L. Ho, "Prepotential approach to solvable rational potentials and exceptional orthogonal polynomials," Prog. Theor. Phys. 126 (2011) 185-201, arXiv:1104.3511[math-ph] .

[18] S. Odake and R. Sasaki, "Krein-Adler transformations for shape-invariant potentials and pseudo virtual states," J. Phys. A46 (2013) 245201 (24pp), arXiv:1212.6595[math$\mathrm{ph}$.

[19] S. Odake and R. Sasaki, "Extensions of solvable potentials with finitely many discrete eigenstates," J. Phys. A46 (2013) 235205 (15pp), arXiv:1301.3980[math-ph].

[20] S. Odake and R. Sasaki, "Crum's theorem for 'discrete' quantum mechanics," Prog. Theor. Phys. 122 (2009) 1067-1079, arXiv:0902.2593[math-ph].

[21] L. García-Gutiérrez, S. Odake and R. Sasaki, "Modification of Crum's theorem for 'discrete' quantum mechanics," Prog. Theor. Phys. 124 (2010) 1-26, arXiv:1004.0289 [math-ph].

[22] S. Odake and R. Sasaki, "Discrete quantum mechanics," (Topical Review) J. Phys. A44 (2011) 353001 (47pp), arXiv:1104.0473[math-ph].

[23] S. Odake and R. Sasaki, "Infinitely many shape invariant discrete quantum mechanical systems and new exceptional orthogonal polynomials related to the Wilson and AskeyWilson polynomials," Phys. Lett. B682 (2009) 130-136, arXiv:0909.3668[math-ph]. 
[24] S. Odake and R. Sasaki, "Exceptional Askey-Wilson type polynomials through DarbouxCrum transformations," J. Phys. A43 (2010) 335201 (18pp), arXiv:1004.0544[math$\mathrm{ph}$.

[25] S. Odake and R. Sasaki, "Exceptional $\left(X_{\ell}\right)(q)$-Racah polynomials," Prog. Theor. Phys. 125 (2011) 851-870, arXiv:1102.0812[math-ph].

[26] S. Odake and R. Sasaki, "Multi-indexed (q-)Racah polynomials," J. Phys. A45 (2012) 385201 (21pp), arXiv:1203.5868[math-ph].

[27] S. Odake and R.Sasaki, "Multi-indexed Wilson and Askey-Wilson polynomials," J. Phys. A46 (2013) 045204 (22pp), arXiv:1207.5584[math-ph].

[28] S. Odake, "Recurrence Relations of the Multi-Indexed Orthogonal Polynomials," J. Math. Phys. 54 (2013) 083506 (18pp), arXiv:1303.5820[math-ph].

[29] D. Gómez-Ullate, Y. Grandati and R. Milson, "Rational extensions of the quantum harmonic oscillator and exceptional Hermite polynomials," arXiv:1306.5143[math-ph].

[30] S. Odake and R. Sasaki, "Casoratian Identities for the Wilson and Askey-Wilson Polynomials," arXiv:1308.4240[math-ph].

[31] E. Routh, "On some properties of certain solutions of a differential equation of the second order," Proc. London Math. Soc. 16 (1884) 245-261; S. Bochner, "Über SturmLiouvillesche Polynomsysteme," Math. Zeit. 29 (1929) 730-736.

[32] G. Darboux, Théorie générale des surfaces vol 2 (1888) Gauthier-Villars, Paris.

[33] M. M. Crum, "Associated Sturm-Liouville systems," Quart. J. Math. Oxford Ser. (2) 6 (1955) 121-127, arXiv:physics/9908019.

[34] M. G. Krein, "On continuous analogue of a formula of Christoffel from the theory of orthogonal polynomials," (Russian) Doklady Acad. Nauk. CCCP, 113 (1957) 970-973; V.É. Adler, “A modification of Crum's method," Theor. Math. Phys. 101 (1994) 13811386.

[35] R. Koekoek and R.F.Swarttouw, "The Askey-scheme of hypergeometric orthogonal polynomials and its q-analogue," arXiv:math.CA/9602214. 
[36] K. Takemura, "Multi-indexed Jacobi polynomials and Maya diagrams," arXiv:1311. $3570[\mathrm{math}-\mathrm{ph}]$. 